(C) 2020 The Author(s) Published by Oxford University Press on behalf of the Royal Astronomical Society. Access to this work was provided by the University of Maryland, Baltimore County (UMBC) ScholarWorks@UMBC digital repository on the Maryland Shared Open Access (MDSOAR) platform.

Please provide feedback

Please support the ScholarWorks@UMBC repository by emailing scholarworks-group@umbc.edu and telling us what having access to this work means to you and why it's important to you. Thank you. 


\title{
Mass outflow of the X-ray emission line gas in NGC 4151
}

\author{
S. B. Kraemer, ${ }^{1 \star}$ T. J. Turner ${ }^{\oplus},{ }^{2}$ J. D. Couto, ${ }^{3}$ D. M. Crenshaw, ${ }^{4}$ H. R. Schmitt,${ }^{5}$ \\ M. Revalski ${ }^{\oplus 6}$ and T. C. Fischer ${ }^{6}$ \\ ${ }^{1}$ Department of Physics, Institute for Astrophysics and Computational Sciences, The Catholic University of America, Washington, DC 20064, USA \\ ${ }^{2}$ Department of Physics, University of Maryland Baltimore County, Baltimore, MD 21250, USA \\ ${ }^{3}$ Department of Physics and Astronomy, Johns Hopkins University, Baltimore, MD 21210, USA \\ ${ }^{4}$ Department of Physics and Astronomy, Georgia State University, 25 Park Place, Room 631, Atlanta, GA 30303, USA \\ ${ }^{5}$ Naval Research Laboratory, Washington, DC 20375, USA \\ ${ }^{6}$ Space Telescope Science Institute, Baltimore, MD 21218, USA
}

Accepted 2020 February 6. Received 2020 February 5; in original form 2019 July 30

\begin{abstract}
We have analysed Chandra/High Energy Transmission Gratings spectra of the X-ray emission line gas in the Seyfert galaxy NGC 4151. The zeroth-order spectral images show extended Hand He-like $\mathrm{O}$ and $\mathrm{Ne}$, up to a distance $r \sim 200 \mathrm{pc}$ from the nucleus. Using the first-order spectra, we measure an average line velocity $\sim-230 \mathrm{~km} \mathrm{~s}^{-1}$, suggesting significant outflow of $\mathrm{X}$-ray gas. We generated CLOUDY photoionization models to fit the first-order spectra; the fit required three distinct emission line components. To estimate the total mass of ionized gas and the mass outflow rates, we applied the model parameters to fit the zeroth-order emission line profiles of Ne IX and NeX. We determined the total mass of $\approx 5.4 \times 10^{5} \mathrm{M}_{\odot}$. Assuming the same kinematic profile as that for the [O III] gas, derived from our analysis of Hubble Space Telescope/Space Telescope Imaging Spectrograph spectra, the peak X-ray mass outflow rate was $\approx 1.8 \mathrm{M}_{\odot} \mathrm{yr}^{-1}$, at $r \sim 150 \mathrm{pc}$. The total mass and mass outflow rates are similar to those determined using [O III], implying that the $\mathrm{X}$-ray gas is a major outflow component. However, unlike the optical outflows, the X-ray outflow rate does not drop off at $r>100 \mathrm{pc}$, which suggests that it may have a greater impact on the host galaxy.
\end{abstract}

Key words: galaxies:active-galaxies: individual: NGC 4151 -galaxies: Seyfert-X-rays: galaxies.

\section{INTRODUCTION}

\subsection{General background}

It is believed that active galactic nuclei (AGNs) are powered by mass accretion on to a supermassive black hole (SMBH) at the gravitational centre of the host galaxy (e.g. Rees 1987). The SMBH and surrounding accretion disc can generate relativistic jets and powerful winds. AGN-driven mass outflows can effect conditions in the host galaxy, in a process referred to as 'AGN feedback' (Begelman 2004). Once the AGN is activated, jets or winds evacuate the bulge of the host galaxy, quenching star formation. This ultimately produces the observed relationship between the mass of the SMBH and the bulge, as evidenced by the $M_{\mathrm{bh}}-\sigma$ relationship (e.g. Gebhardt et al. 2000).

Although radio jets can be powerful enough to drive feedback, only $\sim 15$ per cent of radiation-dominated AGNs are radio-loud (Netzer 2015). On the other hand, the fact that there is evidence that AGN-driven winds are present in $\gtrsim 50$ per cent of AGNs (Ganguly \& Brotherton 2008) suggests that they can span a much larger solid angle and therefore could be the principle feedback mode. Such winds have been detected along our line of sight to AGN in the form of blueshifted absorption lines in high-resolution UV spectra (Crenshaw et al. 1999) and X-ray spectra (Kaastra et al. 2000; Kaspi et al. 2001). Outflow velocities are typically of the order of $100 \mathrm{~s}$ to a few $1000 \mathrm{sm} \mathrm{s}^{-1}$, but can be as large as $0.1-$ $0.5 c$, e.g. in broad absorption line QSOs (e.g. Turnshek 1984) and in AGNs that show highly blueshifted X-ray absorption, i.e. 'Ultra Fast Outflows' (e.g. Tombesi et al. 2011). Nevertheless, in most cases the radial distances and global covering factors of the absorbers are not well constrained, hence the total masses and mass outflow rates and therefore the impact on the host galaxies, are not easily determined.

On the other hand, there has been extensive study of mass outflow in emission in AGNs in the local Universe $(z<0.1)$ (e.g. Fischer et al. 2013). The targets are typically Seyfert galaxies, which are AGNs with modest luminosities, $L_{\mathrm{bol}} \leq 10^{45} \mathrm{erg} \mathrm{s}^{-1}$, although, more recently, Fischer et al. (2018) have extended the study to QSO2s, which are up to several orders of magnitude more luminous. 
While the details of AGN classification have been reviewed numerous times (e.g. Peterson 1997; Netzer 2015), it is useful to briefly summarize them. Generally, AGNs can be classified via their optical emission. Type 1s possess broad permitted lines, with full width at half-maximum $>$ a few $1000 \mathrm{~km}^{-1}$, narrower forbidden lines, with FWHM $\leq 1000 \mathrm{~km} \mathrm{~s}^{-1}$ and non-stellar continua. Type $2 \mathrm{~s}$ have narrow permitted and forbidden lines, and their continua are typically dominated by starlight from the host galaxy. The optical continuum is thought to be thermal emission from the assumed accretion disc. The broad lines are formed in dense gas $\left(n_{\mathrm{H}}>\right.$ $10^{8} \mathrm{~cm}^{-3}$ ) in a region from several to 100 light-days from the central SMBH, referred to as the broad line region (BLR). The narrow components of the permitted lines and the forbidden lines are formed in a region extended from several pcs to several kpcs, called the narrow line region (NLR). The so-called unified model for AGN (Antonucci 1993) postulates that both types are intrinsically the same, but the BLR and continuum source in Type $2 \mathrm{~s}$ are obscured by a dense, molecular torus along our line of sight. The BLR gas is thought to have components from the accretion disc and AGN-driven winds (e.g. Collin et al. 2006), although the winds may include a strong rotational component, if they originate in the disc (e.g. Kashi et al. 2013). The NLR kinematics are consistent with combination of radial outflows and rotation (e.g. Fischer et al. 2017).

NLR-scale outflows in both Type 1 and 2 AGNs have been studied via Hubble Space Telescope (HST)/Space Telescope Imaging Spectrograph (STIS) observations (e.g. Fischer et al. 2013, 2018) and ground-based spectra, such as those obtained with the Gemini/Near Infrared Integral Field Spectrograph (e.g. Storchi-Bergmann et al. 2010; Riffel, Storchi-Bergmann \& Winge 2013; Fischer et al. 2017). The NLR outflows extend to kpc scales (Fischer et al. 2018), where much of the nuclear star formation occurs. Furthermore, most of the mass in these outflows is accelerated in situ, due to the radiation pressure from the AGN accelerating material in the disc of the host galaxy. Hence, the NLR-scale outflows directly reveal the interaction of the AGN with the host galaxy.

Although the NLR outflows are often massive (e.g. mass of $\sim 10^{6}$ $\mathrm{M}_{\odot}$, Collins et al. 2009; Revalski et al. 2018b), and may inject large amounts of kinetic energy and momentum into the interstellar medium (ISM) of the host galaxy (e.g. Revalski et al. 2018a), they do not appear to be able to escape the inner kpc of the host galaxy bulge (Fischer et al. 2018). This calls into question the effectiveness of AGN-driven outflows in feedback. However, these results are generally derived from optical or near-IR emission line kinematics, they do not take into account the role of higher ionization gas.

Low-resolution X-ray spectra of Type 1 AGN, such as those obtained with the Advanced Satellite for Cosmology and Astrophysics (ASCA) revealed absorption by ionized gas along our line of sight to the continuum source (Reynolds 1997), as well as 'spectral complexity' below $<1 \mathrm{keV}$ (George et al. 1998), where the observed continuum emission was weak, due to absorption. Similar soft X-ray properties were detected in ASCA spectra of Type 2 AGN (Turner et al. 1997). Netzer (1993) suggested that the absorber could be a source of the soft X-ray emission and that the spectral complexity would be resolved into emission lines by more sensitive and higher resolution X-ray spectrographs. This prediction was confirmed in observations made with XMM-Newton (Sako et al. 2000; Kinkhabwala et al. 2002; Turner et al. 2003) and Chandra (Ogle et al. 2000). In general, the soft X-ray spectra showed emission lines from $\mathrm{H}$ - and He-like ions of $\mathrm{C}, \mathrm{N}, \mathrm{O}, \mathrm{Ne}$, $\mathrm{Mg}, \mathrm{Si}$, and $\mathrm{S}$, as well as radiative recombination continua (RRC) from those ions.
Chandra X-ray imaging of the Seyfert 2 galaxy NGC 1068 showed that the X-ray emission line gas was extended and coincident with the optical NLR (Young, Wilson \& Shopbell 2001). Bianchi, Guainazzi \& Chiaberge (2006) determined that this was the case for most Seyfert galaxies. They further suggested that the ionization mechanism, specifically photoionization by the AGN, was the same for both the X-ray and optical components. Kallman et al. (2014) performed a detailed photoionization analysis of Chandra/High Energy Transmission Grating (HETG, Canizares et al. 2005) spectra of NGC 1068. They estimated the total mass and mass outflow rates of the emission line gas to be $M \approx 3.7 \times 10^{5}$ $\mathrm{M}_{\odot}$ and $\dot{M}_{\text {out }} \sim 0.3 \mathrm{M}_{\odot} \mathrm{yr}^{-1}$, respectively. Bogdán et al. (2017) analysed Chandra/HETG spectra of the Seyfert 2 galaxy Mrk 3 and derived some constraints on the emission line gas. Although there have been efforts to model the X-ray NLR using Chandra imaging (e.g. Bianchi et al. 2010; Gonzalez-Martin et al. 2010; Maksym et al. 2019), there have not been any other attempts to generate detailed physical models using HETG spectra.

\subsection{Previous study of NGC 4151}

The Seyfert 1.5 galaxy, NGC 4151 ( $z=0.0033$; de Vaucouleurs et al. 1991) has been observed extensively, across most of the electromagnetic spectrum. From reverberation mapping, Bentz \& Katz (2015) derived a black hole mass of $\log M_{\odot}=7.555_{-0.047}^{+0.051}$ The most recent distance estimate is $\approx 15 \mathrm{Mpc}$ (Fausnaugh et al., in preparation), which gives a scale of $70 \mathrm{pc} \mathrm{arcsec}^{-1}$. Most relevant to the work presented here are the results of spatially resolved spectroscopy, obtained with HST/STIS. Using STIS G430M slitless spectra, Hutchings et al. (1998) were the first to unequivocally show that the NLR gas kinematics were consistent with a bi-conical outflow. Kraemer et al. (2000) performed a photoionization analysis of NLR spectra obtained using the STIS G140L, G230L, G430L, and G750L gratings, through the $52 \operatorname{arcsec} \times 0.1$ arcsec aperture. They determined that the NLR gas is photoionized by the EUV$\mathrm{X}$-ray radiation from the AGN. Furthermore, they argued that the gas is inhomogeneous, consisting of co-located radiation and matter bounded components, of different ionization states, and that the gas density decreases with radial distance.

As initially revealed in Chandra/HETG spectra, NGC 4151 possesses soft X-ray emission extending $\sim 1.6 \mathrm{kpc}$ from the nucleus (Ogle et al. 2000). The X-ray emission comprises numerous emission lines from $\mathrm{H}$ - and He-line ions of $\mathrm{C}, \mathrm{N}, \mathrm{O}, \mathrm{Ne}, \mathrm{Mg}$, and Si. Ogle et al. (2000) suggest that, while most of the emission lines are consistent with an origin in a photoionized plasma, there is evidence for collisional enhancement of the Ly $\alpha$ lines of O VIII, $\mathrm{Ne} \mathrm{X}$, and $\mathrm{Mg}$ XII. However, it has been shown that these lines can be strengthened via photo-excitation (e.g. Sako et al. 2000), hence there is no clear signature for a collisional component.

We had previously modelled the soft X-ray emission lines in NGC 4151 using archival XMM-Newton/Reflection Grating Spectrometer observations (Armentrout, Kraemer \& Turner 2007). We measured blueshifted radial velocities for $\mathrm{H}$ - and He-like lines of $\mathrm{C}, \mathrm{N}, \mathrm{O}$ and $\mathrm{Ne}$, with an average value of $\sim-250 \mathrm{~km} \mathrm{~s}^{-1}$. The RRC were consistent with an origin in photoionized gas (Liedahl \& Paerels 1996). The He-like triplets indicated some enhancement of the resonance $1 \mathrm{~s} 2 \mathrm{p}^{1} \mathrm{P}_{1} \rightarrow 1 \mathrm{~s}^{2}{ }^{1} \mathrm{~S}_{0}$ lines (r-lines), compared to the intercombination $1 \mathrm{~s} 2 \mathrm{p}{ }^{3} \mathrm{P}_{2} \rightarrow 1 \mathrm{~s}^{2}{ }^{1} \mathrm{~S}_{0}, 1 \mathrm{~s} 2 \mathrm{p}{ }^{3} \mathrm{P}_{1} \rightarrow 1 \mathrm{~s}^{2}{ }^{1} \mathrm{~S}_{0}$, and forbidden $1 \mathrm{~s} 2 \mathrm{~s}^{3} \mathrm{~S}_{1} \rightarrow 1 \mathrm{~s}^{2}{ }^{1} \mathrm{~S}_{0}$ lines (i- and f-lines). We attributed this to photoexcitation of the r-line (e.g. Sako et al. 2000). We fit the emission line ratios and luminosities with a 3-component photoionization model, generated with the code CLOUDY (for the 
current version, see Ferland et al. 2017). The model values for ionization parameter, $\log U,{ }_{1}^{1}$ and column density, $\log \left(N_{\mathrm{H}} / \mathrm{cm}^{2}\right)$ were $-0.5,20.5 ; 0.0,23$; and, 1.3, 23. We assumed that the emission line gas was associated with the UV and X-ray absorbers discussed in Kraemer et al. $(2005,2006)$ therefore the radial distances were all $<10^{18} \mathrm{~cm}$. Although the models were not physically inconsistent, the RGS data do not possess the spatial resolution to reveal the extent of the emission line gas, hence our analysis did not provide much insight into the mass of ionized gas or mass outflow rates.

Based on their analysis of a $200 \mathrm{ks}$ Chandra/ACIS image of NGC 4151, Wang et al. (2011a, b, c) performed a detailed study of the X-ray emission. In agreement with Ogle et al. (2000), they found X-ray emission in the $0.3-1 \mathrm{keV}$ ('soft') band, extending $30 \mathrm{pc}$ to $1.3 \mathrm{kpc}$, in a north-east (NE) to south-west (SW) direction. The emission cuts off in the NE, at a point coincident with a $\mathrm{CO}$ gas lane in the host galaxy. On the other hand, the 1-7 keV emission appeared unresolved. The soft emission within 2 arcsec of the nucleus shows a linear structure, and there are discrete knots within 1 arcsec. By isolating regions of the spectrum, they were able to map the O VII, O VIII, and Ne IX emission, which appeared spatially correlated with the optical [O III] $\lambda 5007$ emission detected by HST/WFPC2 (see Kraemer, Schmitt \& Crenshaw 2008). They assumed that the $[\mathrm{O} \mathrm{III}]$ and $\mathrm{X}$-ray emission arise from a single, heterogeneous (in density) photoionized medium. Using the [O III] velocities presented in Crenshaw et al. (2015), and a density law $n_{\mathrm{H}} \propto r^{-2}$, where $n_{\mathrm{H}}$ is the hydrogen number density and $r$ is the distance to the ionizing source, Wang et al. (2011c) obtained a mass outflow rate of $\dot{M}_{\text {out }} \approx 2 \mathrm{M}_{\odot} \mathrm{yr}^{-1}$, at $r=130 \mathrm{pc}$, and a peak kinetic luminosity $L_{\mathrm{KE}} \approx 1.7 \times 10^{41} \mathrm{erg} \mathrm{s}^{-1}$, for the X-ray gas. For comparison, Crenshaw et al. (2015) derived a peak value of $\dot{M}_{\text {out }} \sim 3 \mathrm{M}_{\odot} \mathrm{yr}^{-1}$ at $70 \mathrm{pc}$, and a peak $L_{\mathrm{KE}} \approx 4.3 \times 10^{41} \mathrm{erg} \mathrm{s}^{-1}$ at $90 \mathrm{pc}$, for the optical gas.

In this paper, we will use Chandra/HETG spectra to determine the physical conditions within the X-ray emission line gas. In Section 2, we present the method and results of the spectral and imaging analysis. In Section 3, we present the photoionization analysis of the \pm first-order spectra. In Section 4, we apply the modelling results to the analysis of the extended emission measured using the zeroth-order spectra. Finally, in Section 5, we discuss the dynamics and origin of the X-ray outflow.

\section{CHANDRA OBSERVATIONS}

This paper presents imaging and spectroscopic results from our Chandra observations of NGC 4151 at two epochs during 2014, first presented in Couto et al. (2016). The observations total 241 ks in exposure time. These data were split into two epochs due to constraints in the roll angle alignment to meet the observation goals, i.e. to allow us to have the cross-dispersion direction correctly oriented to observe the extended emission of NGC 4151 (see Ogle et al. 2000).

The first data set, OBSID16089, was an exposure of $172 \mathrm{ks}$, obtained over the period 2014 February 12-14 while the second, OBSID 16090, was an exposure of 69 ks taken 2014 March 8-9. Both observations utilized the HETG grating assembly dispersed on to the S-array of the Advanced CCD Imaging Spectrometer (ACIS). CCDs $\mathrm{S} 1$ to $\mathrm{S} 5$ were used. The HETG consists of a pair of grating

${ }^{1} U=\frac{Q}{4 \pi n_{\mathrm{H}} r^{2} c}$, where, $n_{\mathrm{H}}$ is the hydrogen number density, $r$ is the distance to the ionizing source, $c$ is the speed of light, and the ionizing luminosity, $Q=\int_{v_{o}}^{\infty} L_{v} \mathrm{~d} v$, where $h v_{\mathrm{o}}=13.6 \mathrm{eV}$. arrays. The High Energy grating (HEG) covers $\sim 0.8-8.5 \mathrm{keV}, 16-$ $1.5 \AA$ with a spectral resolution of $0.012 \AA$ FWHM. The medium energy grating (MEG) covers $31-2.5 \AA$ with spectral resolution $0.023 \AA$ AWHM.

Standard level 2 events files were taken as the starting point and Chandra Interactive Analysis of Observations (CIAO) software version 4.10 together with CALDB 4.7.9 and HEASOFT v6.24 were used for the data analysis. The mean source flux for the 2014 observations was $F_{2-10}=6.3 \times 10^{-11} \mathrm{erg} \mathrm{cm}^{-2} \mathrm{~s}^{-1}$. The total number of counts in the summed (positive and negative) first-order HEG spectra were 35938 counts, with a corresponding count rate $0.1483 \pm 0.0010$ count $\mathrm{s}^{-1}$ and, for MEG, 32115 counts, with a count rate of $0.1326 \pm 0.0009$ count $\mathrm{s}^{-1}$. The background level was negligible compared to the source. The full, unfolded spectrum is shown in Fig. 1.

\subsection{Imaging analysis}

Based upon previous spectral and imaging analysis of NGC 4151 (e.g. Ogle et al. 2000; Wang et al. 2011a), we selected 12 wavebands that allowed us to study the spatial dependence at wavelengths dominated by emission lines of interest. We did not subtract the continuum from the images. Two line-free continuum bands were also chosen for comparison with the emission line maps.

The two OBSIDs were combined for imaging analysis, using CIAO task merge_obs. This task creates an exposure map for each observation that accounts for the dither pattern and allows correct summation of observations with different roll angles. This yielded images in units of photons $\mathrm{cm}^{-2} \mathrm{~s}^{-1}$. The images created were sampled at the maximum spatial resolution of ACIS, i.e. with pixels of $\sim 0.5 \operatorname{arcsec}$ on a side. These images were smoothed with a Gaussian having a 1 arcsec kernel radius. The colour maps were adjusted to show the same flux scale for each image.

As shown in Fig. 2, the continuum, both at 2.05-2.25 and 5.60$6.00 \mathrm{keV}$, is roughly symmetric and centred on the nucleus. The higher flux in the higher energy bin is the result of lower absorption than at lower energies (see also Fig. 1). There is some evidence for weak emission in the $2.05-2.25 \mathrm{keV}$ band, towards the NE, but its origin is uncertain. The $\mathrm{Si} \mathrm{K} \alpha$ and $\mathrm{Fe} \mathrm{K} \alpha$ lines are also strongly centrally peaked, again with a slight extension to the NE. However, the location of the peak of the emission encompasses the innermost region of the AGN, hence most of the $\mathrm{K}$ line emission is consistent an origin in the putative torus. As such, we have modelled it as due to reflection (see below).

The lines with energies $>1.3 \mathrm{keV}$, e.g. Si XIV, Si XIII, and Mg XII, are all quite compact, in agreement with Wang et al. (2011a) regarding the morphology of the emission in the $1-7 \mathrm{keV}$ band. On the other hand, there is evidence that $\mathrm{Mg} \mathrm{XI}$ is extended along the NE-SW direction. Lines with energies $\leq 1.05 \mathrm{keV}$, i.e. NeX, Ne IX, O VIII, and O VII, are all clearly extended along a NE-SW direction, out to a projected distance of $>200 \mathrm{pc}$. Although this is smaller than that noted by Wang et al. (2011a, b), it is not unexpected considering the lower effective area of the HETG and the loss in ACIS sensitivity at energies $<1 \mathrm{keV}$ (O'Dell et al. 2013). We revisit the nature of this extended emission in Section 4.

\subsection{Global spectral analysis}

We first analysed spectra over the entire NLR, from which we obtained emission line and RRC fluxes and line velocity centroids. Spectra were extracted using the default extraction criteria and were pulled from the PHA2 files provided with the pipeline products for 


\section{Unfolded Spectrum}

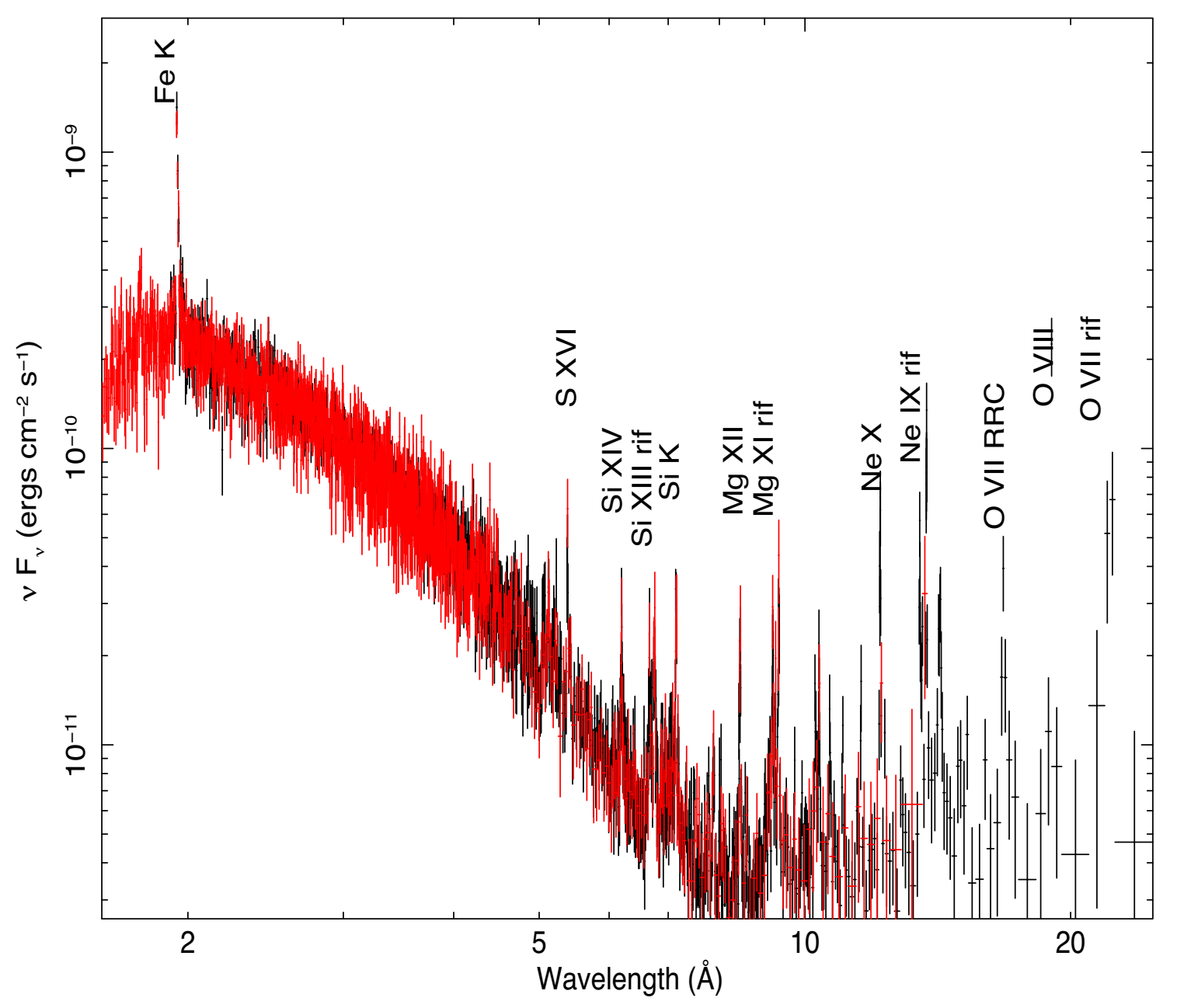

Figure 1. Full, \pm first-order, HETG spectrum from 1.59-24.8 , from the summed OBSIDs (see Section 2.2). The red and black points are from the HEG and MEG, respectively. The data were binned to a minimum of 10 counts (per bin). The turn-over towards lower energies (greater wavelengths) is due to intrinsic absorption (see Couto et al. 2016). Emission at wavelengths $>6 \AA$ is emission line dominated.

each OBSID. ARF and RMF files were generated for each OBSID, then the pairs of positive and negative spectra were combined for the first-order spectra. The data were binned to a minimum of 10 counts per spectral bin such that the $\chi^{2}$ statistic could be utilized (e.g. Arzner et al. 2007). However, in order to determine whether our results were biased by our binning limit, we also fit the data binned to a minimum of 20 counts per spectral bin. The results of the test are discussed in Appendix A. In summary, there were no significant differences, except that the coarser binning washed out several emission features. Hence, we have opted to use the 10 count minimum for our analysis. The fits were performed with the positive and negative parts of the spectra kept separate. The two OBSIDs were fitted jointly, rather than being co-added. For the spectral plots we combined the positive and negative orders, with both OBSIDs co-added, and applied additional binning, for clarity in the figures.

The hard energy band of the spectrum is dominated by nuclear continuum emission, and the prominent $\mathrm{Fe} \mathrm{K} \alpha$ line is evident, at $6.4 \mathrm{keV}(\lambda=1.94 \AA)$. At energies $<2 \mathrm{keV}$, there are strong emission lines from $\mathrm{H}$ - and $\mathrm{He}-$ like $\mathrm{O}, \mathrm{Ne}, \mathrm{Mg}$, and $\mathrm{Si}$, and there is a $\mathrm{Si} \mathrm{K} \alpha$ line, at $1.74 \mathrm{keV}(\lambda=7.12 \AA)$.

\subsubsection{Emission line and RRC measurements}

For the lines, we fitted a Gaussian model and local continuum (using a power law, with an un-constrained slope) for a restricted bandpass close to the line, using $\chi^{2}$ statistics. The width of the bandpass used depended on the particular region, i.e. whether it was crowded or the line was isolated. The line flux and observed energy were then fitted for most lines. In most cases, the line widths were frozen at $2 \mathrm{eV}$, because the lines were generally too weak to determine width. The RRCs were fitted using the XSPEC model 'redge', with local continuum fitted as a simple power law, as for the lines. The RRC energies and widths were fixed. Subsequently, we fit for the RRC fluxes.

The resulting emission line and RRC fluxes are listed in Table 1. For those lines which were detected both in the HETG and $X M M /$ RGS spectra (Armentrout et al. 2007), i.e. Ne IX rif, NeX $\alpha$, O VII rif, and $\mathrm{O}$ VIII $\alpha$, the measured fluxes agree, within the uncertainties. We were not able to obtain accurate widths, or corresponding temperatures, for any of the RRCs. However, as we will show in Section 3.2, the photoionization model predictions for the $\mathrm{O}$ VII and $\mathrm{O}$ VIII RRCs are consistent with the data. 

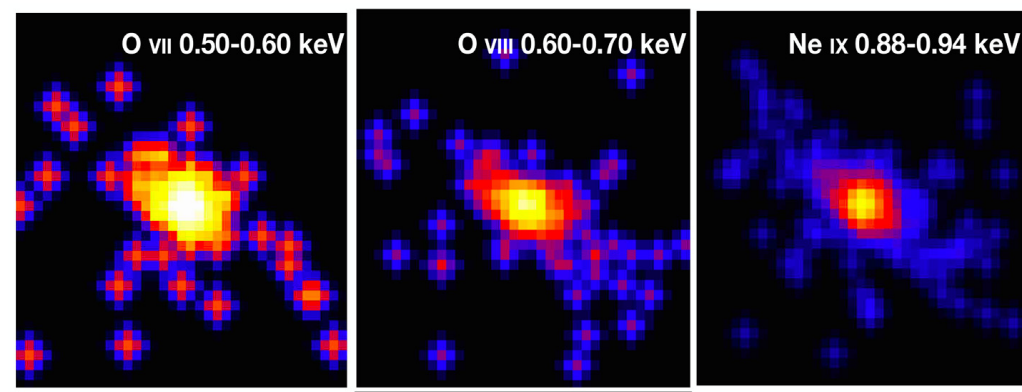

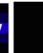

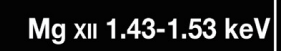

Si I 1 1.734-1.74 keV
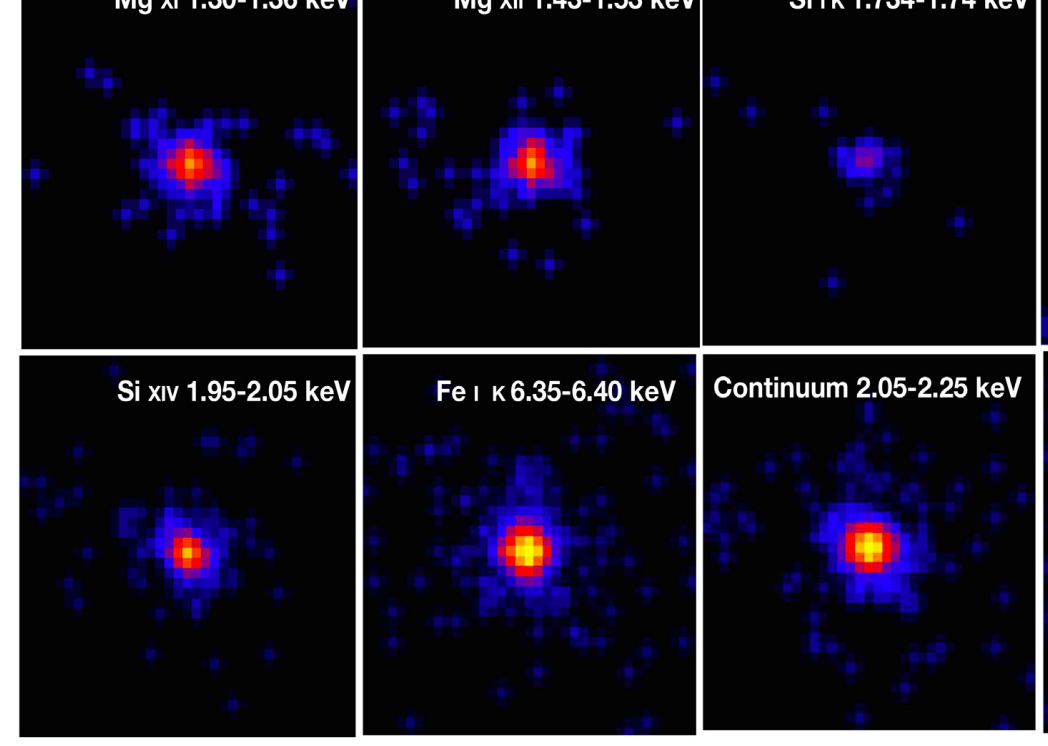
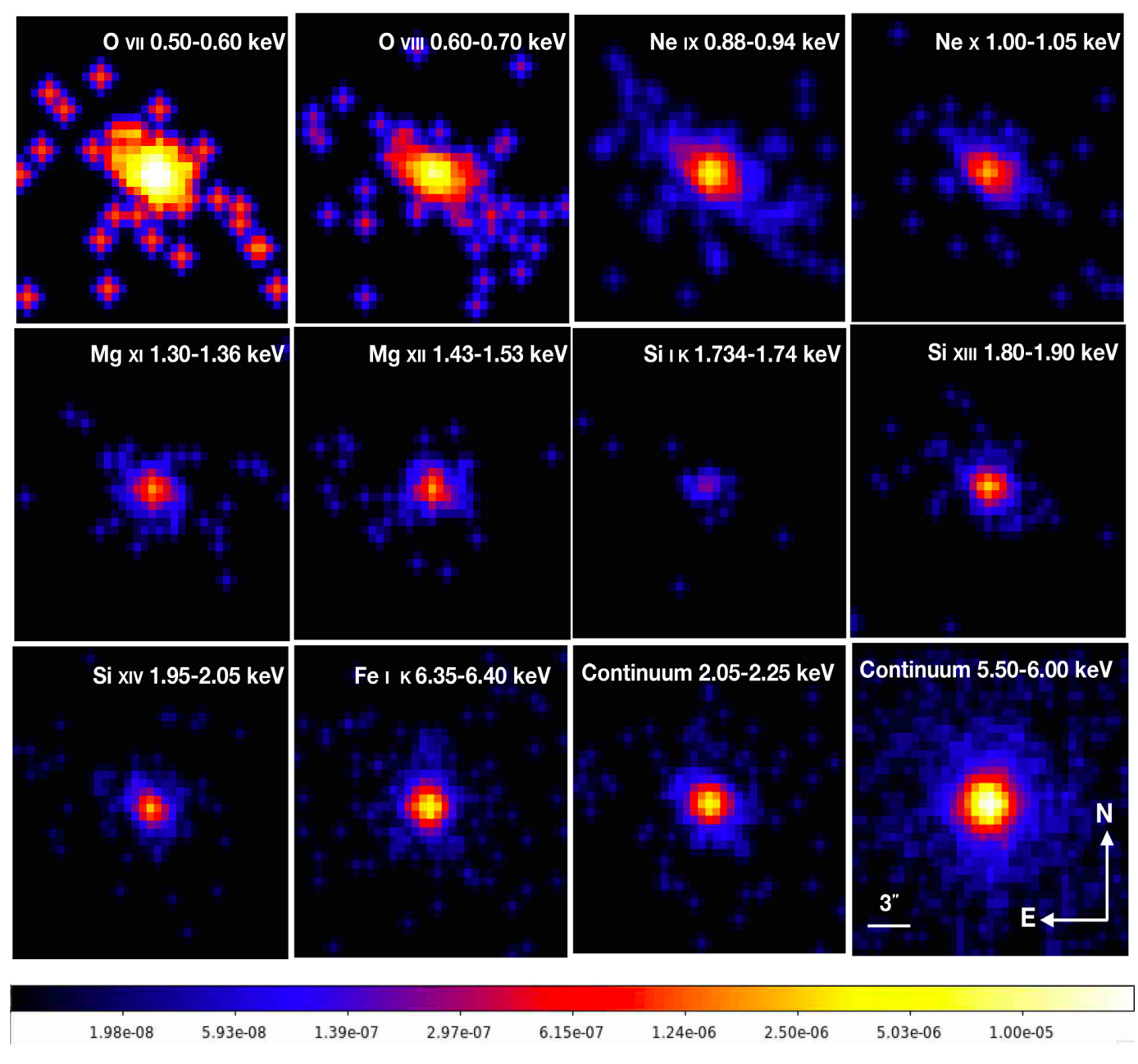

$1.24 \mathrm{e}-06$

$2.50 \mathrm{e}-06$

5.03e-06 $\quad 1.00 \mathrm{e}-05$

Figure 2. Emission profiles for hydrogen and helium-like $\mathrm{O}, \mathrm{Ne}, \mathrm{Mg}$, and $\mathrm{Si}$ lines, and $\mathrm{Si} \mathrm{K} \alpha$ and $\mathrm{Fe} \mathrm{K} \alpha$ lines, from the zeroth-order HETG spectra. Line-free regions of continuum in the ranges 2.05-2.25 and 5.50-6.00 keV are also shown. The images have been smoothed with a Gaussian function with kernel radius of 1 arcsec, and are equally scaled for comparison in the colourbar in terms of total number of counts. The spatial scale and orientation are shown in the lower right panel.

Due to the structure of the underlying continuum, the statistical significance of the emission features must be evaluated in the context of the overall fitting of the data. We will address this in Section 3.1.

\subsubsection{Emission line velocities}

In Table 1, we also list the radial velocities for the strongest emission lines. In Fig. 3, we plot the velocities versus ionization potential (IP); given the uncertainties in the velocities for Ne IX f, O VII f, and O VIII $\alpha$, we used the values in Armentrout et al. (2007), as well as their velocities for N VII $\alpha$ and N VI f, which were not detected in the HETG spectra. The average radial velocity is $v_{\mathrm{r}}=-230_{-370}^{-90} \mathrm{~km}$ $\mathrm{s}^{-1}$. Although there is evidence for a relationship between velocities and the IP for optical and UV lines in some Seyfert galaxies (Kraemer \& Crenshaw 2000; Kraemer et al. 2009), which suggests a multicomponent NLR, we found no strong evidence of such an relationship in NGC 4151 (Kraemer et al. 2000). Therefore, while the X-ray emission line gas consists of components of different ionization, as we show in Section 3.2, there is no evidence for kinematic differences among them, which is consistent with the optical and UV result.
The X-ray emission lines velocities were measured using spectra covering the entire region detected in these observations. Hence, they are a flux-weighted average. For comparison, Crenshaw et al. (2010) derived an integrated [O III] emission line profile by collapsing HST/STIS G430M, which spanned most of the NLR. They obtained $v_{\mathrm{r}}=-60( \pm 6) \mathrm{km} \mathrm{s}^{-1}$. However, their measurements included redshifted emission on the NE side of the nucleus. The emission line gas in that region lies behind the disc of the host galaxy (Das et al. 2005) and could be covered by X-ray absorption similar to that described by Couto et al. (2016). In fact, soft X-ray emission lines can be effectively suppressed by relatively small column density absorbers (e.g. $N_{\mathrm{H}} \leq 10^{21} \mathrm{~cm}^{-2}$, Kraemer et al. 2011). If the difference in $v_{\mathrm{r}}$ is due to suppression of the X-ray emission in the NE, it is highly plausible that the X-ray gas kinematics are similar to that of the [O III] gas. We revisit this in Section 4.2.

\section{MODELLING ANALYSIS}

\subsection{Inputs to the models}

Our principle goal for this analysis is to determine the physical properties of the global, i.e. unresolved, emission. To that end, we 
Table 1. Measured emission features.

\begin{tabular}{|c|c|c|c|c|c|c|}
\hline Ion & $\begin{array}{c}\text { Ionization potential }^{a} \\
\qquad(\mathrm{keV})\end{array}$ & $\begin{array}{l}\text { Wavelength } \\
(\AA)\end{array}$ & $\begin{array}{c}\text { Flux } \\
\left(\times 10^{-6} \mathrm{~cm}^{-2} \mathrm{~s}^{-1}\right)\end{array}$ & $\begin{array}{c}\sigma^{b} \\
(\mathrm{eV})\end{array}$ & $\begin{array}{c}v_{\mathrm{off}^{\mathrm{c}}} \\
\left(\mathrm{km} \mathrm{s}^{-1}\right)\end{array}$ & $\Delta \chi^{2 d}$ \\
\hline $\operatorname{SixIV} \alpha$ & 2.44 & $6.202_{-0.005}^{+0.005}$ & $10.0 \pm 1.0$ & $1.0-5.2$ & $-156_{-375}^{+84}$ & -28 \\
\hline $\mathrm{Si} X I I I \mathrm{r}$ & 0.52 & 6.666 & $5.4 \pm 0.7$ & & & $-118^{e}$ \\
\hline Si XIII i & & 6.707 & $2.6 \pm 0.4$ & & & \\
\hline Si XIII f & & $6.758_{-0.006}^{+0.001}$ & $8.1 \pm 1.4$ & $<5.9$ & $-217_{-463}^{-146}$ & \\
\hline $\operatorname{Mg}$ XII $\beta$ & 1.76 & 7.122 & $4.6 \pm 0.7$ & $<0.4$ & & -8 \\
\hline $\operatorname{Mg}$ XII $\alpha$ & & $8.446_{-0.003}^{+0.005}$ & $7.4 \pm 0.8$ & & $-149_{-255}^{+12}$ & -28 \\
\hline Ne X RRC & 1.20 & 9.17 & $12.1 \pm 2.7$ & & & -3 \\
\hline $\operatorname{Mg}$ XI r & 0.37 & 9.193 & $7.4 \pm 1.1$ & & & $-25^{e}$ \\
\hline $\mathrm{Mg} X \mathrm{XI}$ & & 9.258 & $3.1 \pm 0.9$ & & & \\
\hline $\operatorname{Mg}$ XI f & & $9.337_{-0.007}^{+0.006}$ & $10.0 \pm 1.5$ & & $-244_{-452}^{-37}$ & \\
\hline Ne IX RRC & 0.24 & 10.37 & $5.6 \pm 1.0$ & & & -30 \\
\hline $\operatorname{Ne} \mathrm{X} \alpha$ & & $12.169_{-0.009}^{+0.003}$ & $29.5 \pm 3.0$ & & $-284_{-353}^{-66}$ & -40 \\
\hline Ne IX r & & 13.50 & $25.2 \pm 6.0$ & & & $-45^{e, f}$ \\
\hline Ne IX i & & 13.60 & $23.1 \pm 6.0$ & & & \\
\hline NeIX $f$ & & 13.73 & $72.5 \pm 7.0$ & $<1.0$ & -295 (fixed) & \\
\hline O VIII RRC & 0.74 & 14.25 & $42.0 \pm 6.0$ & & & \\
\hline O VII RRC & 0.14 & 16.82 & $56.0 \pm 13.0$ & & & \\
\hline O VIII $\alpha$ & & $19.05_{-0.13}^{+0.09}$ & $104.0 \pm 19.0$ & $<1.0$ & $+231_{-1852}^{+1730}$ & \\
\hline O VII r & & 21.71 & $72.3 \pm 43.0$ & & & \\
\hline O VII f & & $22.19_{-0.01}^{+0.03}$ & $378.0 \pm 85.0$ & $0.4-2.0$ & $-236_{-412}^{+199}$ & \\
\hline
\end{tabular}

${ }^{a}$ Ionization potentials from Cox (2000).

${ }^{b}$ Line width, in $\mathrm{eV}$, derived from a Gaussian fit to the line. When blank, the widths were fixed at $2 \mathrm{eV}$, for Gaussian lines, and $k T_{\mathrm{e}}=5 \mathrm{eV}$ for RRCs, where $k$ is Boltzmann's constant and $T_{\mathrm{e}}$ is the electron temperature.

${ }^{c}$ Blank fields indicate no constraints were obtained.

${ }^{d}$ Significance of feature returned by the sliding Gaussian test (Section 3.1; Fig. 4). Features at $\lambda>15 \AA$ were not tested, due to the weakness of the continuum.

${ }^{e}$ Combined for the full triplet.

${ }^{f}$ Combined with O VIII RRC.

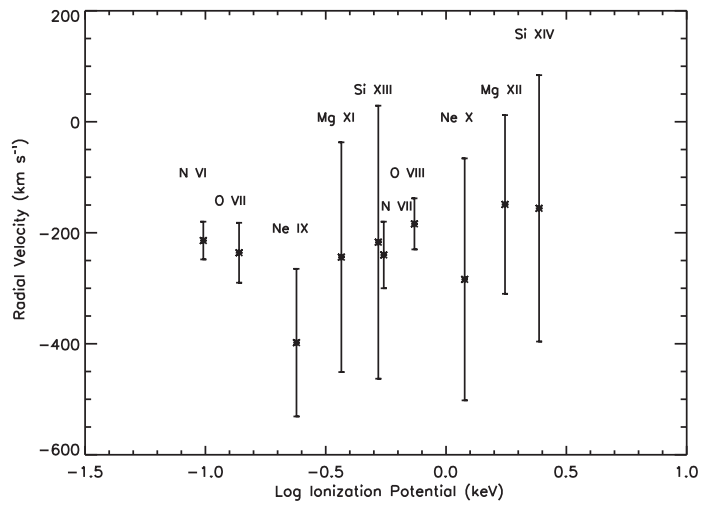

Figure 3. Radial velocities plotted versus IP. Velocities of H-like ions were measured from their Ly $\alpha$ transitions, while those for the He-like ions were measured from the triplet forbidden lines. Velocities from N VI, N VII, O VII, $\mathrm{O}$ VIII, and Ne IX were taken from the $X M M /$ RGS analysis (Armentrout et al. 2007). IPs not listed in Table 1 are N VI, IP $=0.098 \mathrm{keV}$; N VII, IP $=0.55 \mathrm{keV}$ (Cox 2000).

generated photoionization model grids, computed using CLOUDY (version 13.00; Ferland et al. 2013 ${ }^{2}$ ). We assumed a continuum source with the same spectral energy distribution (SED) used in our analysis of the X-ray absorbers (Couto et al. 2016), in the form of a power law $L_{v} \propto v^{-\alpha}$, with $\alpha=1.0$, for $1 \times 10^{-4}$ $\mathrm{eV}<h v<13.6 \mathrm{eV}, \alpha=1.3$, for $13.6 \mathrm{eV}<h v<500 \mathrm{eV}$, and $\alpha=$

${ }^{2}$ The models were rerun with the C17 version of CLOUDY (Ferland et al. 2017), with no significant differences in the model results.
0.5 , for $500 \mathrm{eV}<h v<30 \mathrm{keV}$, with exponential cut-offs above and below the limits. In addition to the SED, the model results depend on the choice of input parameters, specifically: the radial distances of the emission line gas with respect to the central source $(r)$, number density $\left(n_{\mathrm{H}}\right)$, and column density $\left(N_{\mathrm{H}}\right)$ of the gas. The models are parametrized in terms of the ionization parameter, $U$.

The continuum emission in NGC 4151 is highly variable, from optical to X-ray energies (e.g. Edelson et al. 1996). For our models, we scaled the ionizing luminosity, $Q$, using a rough average UV flux at $1350 \AA$ observed over the last $\sim 20$ yr (see fig. 3 in Couto et al. 2016), and computed $Q$ using our assumed SED and a distance of $15 \mathrm{Mpc}$. We obtained a value $Q=3 \times 10^{53}$ photons $\mathrm{s}^{-1}$. For comparison, using the same SED, Wang et al. (2011b) determined a value of $Q=7.2 \times 10^{53}$ photons s $^{-1}$, while Couto et al. (2016) used $Q=0.4-1.1 \times 10^{53}$ photons s $^{-1}$ to model the intrinsic absorption. Based on this continuum, the bolometric luminosity $L_{\mathrm{bol}}=1.4 \times 10^{44} \mathrm{erg} \mathrm{s}^{-1}$ or 0.031 of the Eddington luminosity.

The elemental abundances in NLR emission line gas in NGC 4151 were initially determined in our photoionization modelling of STIS long slit spectra (Kraemer et al. 2000). Based on more recent estimates of solar elemental abundances (e.g. Asplund, Grevesse \& Sauval 2005), the abundances we had determined for NGC 4151 correspond to roughly 1.5 times solar. We assumed these abundances in our most recent warm absorber study of NGC 4151 (Couto et al. 2016), and have opted to assume the same values for this paper, as follows (in logarithm, relative to $\mathrm{H}$, by number): $\mathrm{He}$ : $-1.00, \mathrm{C}:-3.47, \mathrm{~N}:-3.92, \mathrm{O}:-3.17, \mathrm{Ne}:-3.96, \mathrm{Na} ;-5.76, \mathrm{Mg}$ : $-4.48, \mathrm{Al}:-5.55, \mathrm{Si}:-4.51, \mathrm{P}:-6.59, \mathrm{~S}:-4.82, \mathrm{Ar}:-5.60, \mathrm{Ca}$ : $-5.66, \mathrm{Fe}:-4.4$, and $\mathrm{Ni}:-5.78$. 
Table 2. Fit parameters for the HETG data. ${ }^{a}$

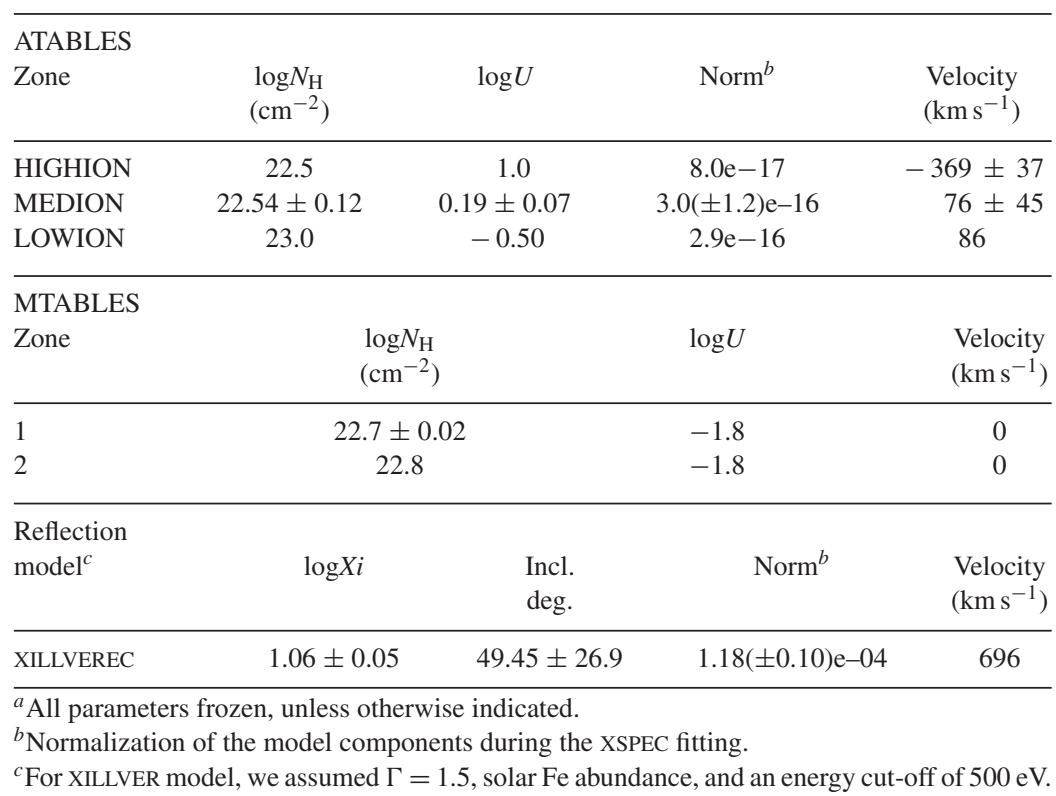

We generated grids of photoionization models over a range of values of $U$ and $N_{\mathrm{H}}$, using the default energy resolution. We converted the CLOUDY output to fittable grids using the Porter et al. (2006) CLOUDY-to-XSPEC interface, which maps the CLOUDY output on to a FITS format grid of either the additive emission components (ATABLES) or multiplicative absorption coefficients (MTABLES). XSPEC (Arnaud 1996) works by interpolating between grid values in the fitting process.

The X-ray spectrum of NGC 4151 is complex, comprising several components: (1) heavily absorbed power-law continuum emission from the AGN, (2) reflection, including the Fe K and Si K fluorescence lines, (3) emission lines and RRCs, and (4) scattered or unabsorbed power-law continuum. The counts in the data are dominated by the first two of these (see Fig. 1). Therefore, our approach was to obtain a satisfactory fit to the absorbed powerlaw and reflection components. Next, we added ATABLES for the emission features, other than those associated with reflection, e.g. the $\mathrm{Fe} \mathrm{K}$ and $\mathrm{Si} \mathrm{K}$ lines. Since the emission features are weak compared to the continuum, the addition of the ATABLES had a negligible effect on the fit statistics. Nevertheless, once we had a good fit to the underlying continuum, we were able to test for the statistical significance of the emission features, as we describe below. Then, we adjusted the parameters of the ATABLES to optimize the fit to the statistically significant emission lines and RRCs. In the process, we froze selected parameters from the earlier iterations; the final model parameters are listed in Table 2.

In the analysis, we used data over $0.9-7.8 \mathrm{keV}(13.78-1.59 \AA)$, for the HEG, and 0.5-6.0 keV (24.8-2.07 $\AA$ ), for the MEG. To model the low-ionization reflection we used the XILLVEREC model, version 1.2.0 (Dauser et al. 2013; García et al. 2013). All models included the Galactic line-of-sight absorption, $N_{\mathrm{H}, \mathrm{Gal}}=2.0 \times 10^{20} \mathrm{~cm}^{-2}$ (Dickey \& Lockman 1990), parametrized using TBABS.

The ATABLE grid spanned a range from $22 \leq \log N_{\mathrm{H}} \leq 23$ and $-0.5 \leq \log U \leq 1.3$, in intervals of $0.1 \mathrm{dex}$; the ranges are consistent with those used in Armentrout et al. (2007). We also assumed a turbulence velocity of $100 \mathrm{~km} \mathrm{~s}^{-1}$, in agreement with our previous studies (Armentrout et al. 2007; Couto et al. 2016). The MTABLES were generated via a two-step process. First, we generated a single zone, with parameters, $\log U=0.42$ and $\log N_{\mathrm{H}}=$ 22.7, to match the high-ionization absorber, XHIGH, described in Couto et al. (2016). Then, using the continuum transmitted through $\mathrm{XHIGH}$, we generated an MTABLE grid spanning a range from $22.4 \leq \log N_{\mathrm{H}} \leq 22.8$ and $-1.8 \leq \log U \leq 0.18$, in intervals of 0.1 dex. The rationale for the filtering of the continuum is explained in Kraemer et al. (2005).

The baseline model comprised a power-law continuum, whose slope reached a preferred value $\Gamma=1.50 \pm 0.04$, consistent with the SED used for the CLOUDY models. This power law was absorbed by two zones (MTABLES), the first with 100 per cent covering of the continuum source and the second covering the first zone. Inclusion of the two zones improved the fit from a reduced $\chi^{2}=5.92$ for 3054 degrees of freedom (dof), to 1.26 , for 3053 dof. The addition of the XILLVEREC model improved the fit to a reduced $\chi^{2}=1.03$ for 3053 dof. While the inclusion an unabsorbed power law, at 3 percent of the flux of the continuum source, made a small statistical change $\left(\Delta \chi^{2}=158\right)$, visual inspection showed improvement of the continuum fitting, consistent with the suggestion by Kraemer et al. (2005). The overall continuum fit was optimized to isolate the emission features, hence, while roughly consistent with Couto et al. (2016), we did not require it to be an exact match.

Having obtained a good fit to the absorbed power-law plus reflection, we were now able to assess the significance of the emission features. To do so, we executed a sliding Gaussian test (see Turner et al. 2003). Specifically, for each detector, we stepped a Gaussian profile across the spectra in $0.1 \AA$ intervals. The Gaussian width, expressed as $\sigma$, was always fixed at $2 \mathrm{eV}$. The line was set to the first wavelength and fixed, then fit for flux and a $\Delta \chi^{2}$, showing the improvement to the fit for a narrow Gaussian being added at that energy, is returned. Then, the Gaussian was moved one step to next wavelength, and the process was repeated. Thus, for each fit step, the line wavelength and width are fixed and the data were tested for the presence of a significant narrow line. Note, when this test encounters a broad feature, e.g. an RRC or an unresolved He-like triplet, it returns a broad hump in $\Delta \chi^{2}$, as the Gaussian first encompasses the wings of the feature and, then, the peak, as it sweeps over it. 

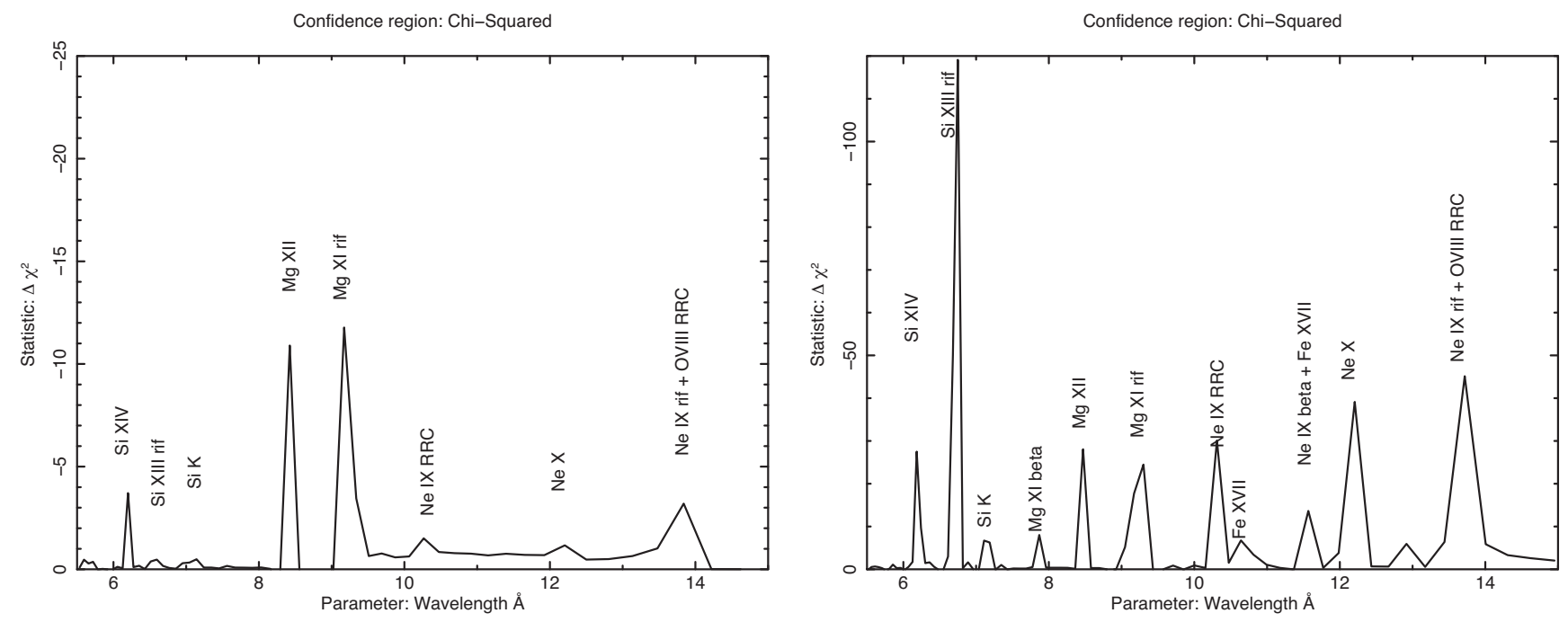

Figure 4. Sliding Gaussians tests, over the range 5.5-15 ̊, for the HEG (left-hand panel) and MEG (right-hand panel) spectra. The test was performed on the spectra with the absorbed power-law and reflection components included (see Section 3.1). The detected emission features are labelled.

The results of the sliding Gaussian are shown in Fig. 4. Note, we did not extend the test to wavelengths $<5.5 \AA$, since we did not detect strong emission lines in that region other than Fe K. The emission features listed in Table 1 are all present. Those emission features used to constrain the models were those that returned values of $\Delta \chi^{2}$ of more than -25 , although we list the those with weaker statistical significance to compare to the CLOUDY predictions (see below). We did not test to wavelengths $>15 \AA$, since the signal to noise of the spectra were too low in that region for this method to return accurate results. However, as shown in Fig. 2, O VIII and the $\mathrm{O}$ VII rif lines are clearly present in the spectra, hence we included them in our models. We next added the ATABLES to fit the detected emission features.

As discussed in Section 2, there are emission lines over a wide range in IP in the HETG spectra. To fit these, we included three zones (ATABLES), which we refer to as HIGHION, MEDION, and LOWION. MEDION produces most of the emission lines and therefore we were able to constrain its parameters via the XSPEC fitting. However, the fewer emission lines from the other components, coupled with the weakness of the line fluxes compared to even the heavily absorbed power law, made it impossible to constrain their parameters. Hence, once we had obtained a good fit for MEDION, we compared the line fluxes predicted by the CLOUDY models to our measured fluxes to estimate $U$ and $N_{\mathrm{H}}$ for HIGHION and LOWION. Then we added those components to optimize the fit to the emission lines, with the constraint that we could not overpredict features. The final fitted parameters are listed in Table 2 and the individual model components are shown in Fig. 5. Unless otherwise stated, parameters in Table 2 are quoted in the rest frame of the source and errors are at the 90 per cent confidence level for one interesting parameter $\left(\Delta \chi^{2}=2.706\right)$. For the final model, the reduced $\chi^{2}=1.028$, however, as noted above, the statistics were dominated by the continuum fitting, rather than the emission lines or RRCs.

The values of $U$ and $N_{\mathrm{H}}$ for HIGHION and MEDION are similar to the two highest ionization components used to fit the $X M M / \mathrm{RGS}$ spectra (Armentrout et al. 2007). LOWION has the same $U$ as the lowest ionization component in Armentrout et al. (2007), but a significantly larger $N_{\mathrm{H}}$. At $\log U=-0.5$, the strongest emission lines, such as the O VII triplet, are formed near the ionized face of the slab, hence the fit to those lines is not sensitive to the column density. However, there is some emission from inner shell $\mathrm{Si} \mathrm{X-Si} \mathrm{V,}$ in the 6.85-6.92 $\AA$ range (see Fig. 6). These arise in LOWION, but would not have been detected in the RGS spectra, which is likely why the value of $N_{\mathrm{H}}$ in (Armentrout et al. 2007) was so much lower. In any case, given the quality of these data and the limited contribution from LOWION, its overall physical properties cannot be tightly constrained.

\subsection{Initial model results}

In Figs 6 and 7, we compare the complete model to the HEG and MEG spectra, with the emission features listed in Table 1 identified. In general, there is good agreement between the model and the data, in the sense that all of the strong emission lines and RRCs are present in the model. The only major discrepancy is in the region near the Si XIII triplet, at 6.6-6.8 $\AA$ in the observed frame. In particular, there appears to be un-modelled emission at $\sim 6.7 \AA$. In their analysis of the HETG spectra of the Seyfert 2 galaxy NGC 1068, Kallman et al. (2014) identified this as Mg XII, but the $\mathrm{Mg}$ XII $\beta$ line is relatively weak in their data, as well as ours (see Table 1), and this is at too low energy to be the Mg XII RRC. Therefore, the source of the emission is unclear.

Although, overall the fit is reasonable, the model unpredicts many of the emission lines. This can be rectified by increasing the nomalization of the ATABLES, but to do so forces the model continuum to lie above the observed continuum. The limitation of the modelling is likely due to unmodelled structure in the continuum. For example, as shown in Fig. 6, in the range from 7 to $8.5 \AA$, the model flux lies above the data, and the latter shows structure consistent with unmodelled absorption. However, there is no obvious need for additional emission components, or a broader range in $U$ and $N_{\mathrm{H}}$ for the ATABLES.

\subsubsection{Distance and density constraints}

The ATABLE grid was generated assuming a single density, $n_{\mathrm{H}}=10^{5} \mathrm{~cm}^{-3}$. We can achieve better constraints on the model parameters by requiring $\Delta r / r<1$ for each component, where $\Delta r$ and $r$ are the physical depth and distance from the ionzing source, 

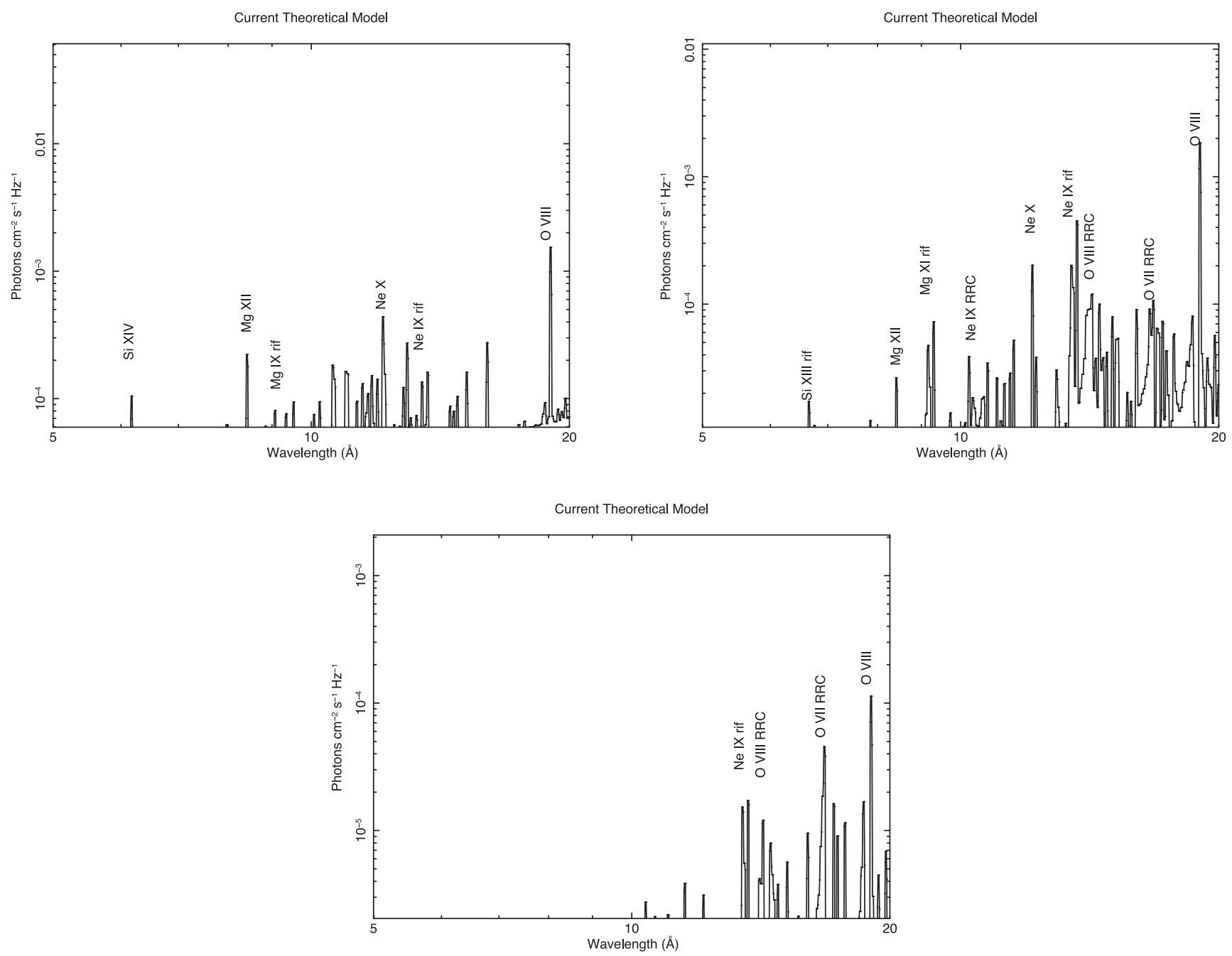

Figure 5. Predicted emission line spectra in the range 5-20 ̊, for the three model components described in the text: HIGHION (left-hand panel), MIDION (right-hand panel), and LOWION (lower panel). The plots were generated from ATABLES created with the CLOUDY model grid. Emission features used in the model fitting are labelled.
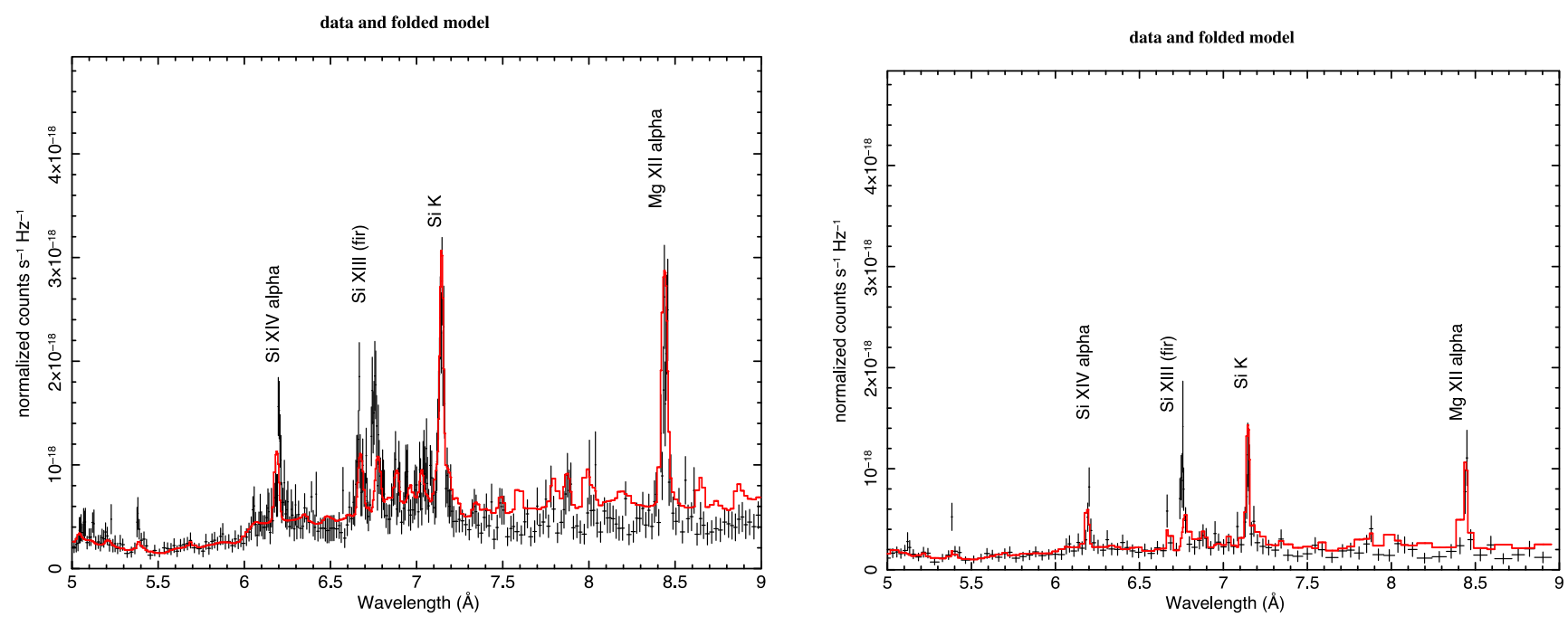

Figure 6. Left-hand panel: Full model, in red, plotted against the MEG data, in black, for the range 5-9 A. Strong emission features are identified. Overall, the model provides an adequate fit to the data, albeit with the discrepancies discussed in Section 3.2. Right-hand panel: Full model, in red, plotted against the HEG data, in black, for the range 5-9 A. 

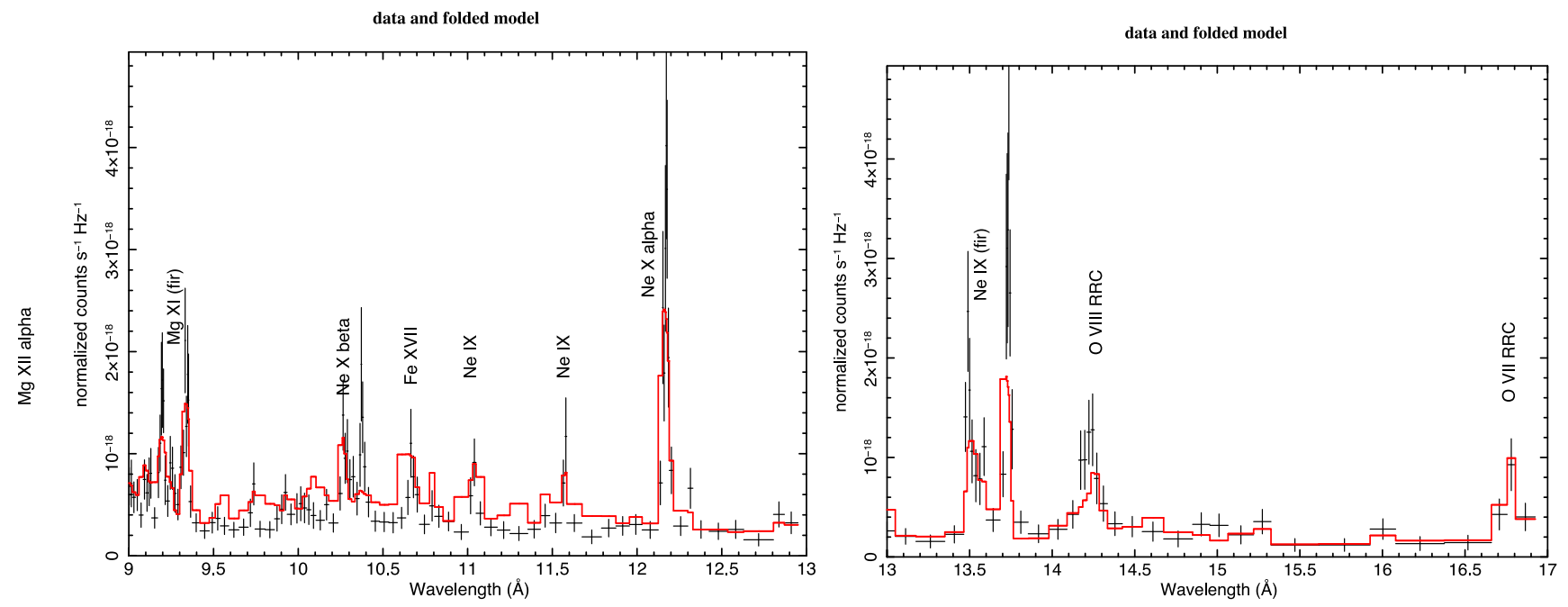

Figure 7. Left-hand panel: Full model (red) versus MEG data (black) for the range 9-13 Å. Right-hand panel: Same for the range 13-17 A.

Table 3. CLOUDY model parameters. ${ }^{a}$

\begin{tabular}{lccccr}
\hline Model & $\begin{array}{c}\log \left(n_{\mathrm{H}}\right) \\
\left(\mathrm{cm}^{-3}\right)\end{array}$ & $\begin{array}{c}\text { Distance } \\
(\mathrm{cm})\end{array}$ & $\begin{array}{c}A \\
\left(\mathrm{~cm}^{2}\right)\end{array}$ & $C_{\mathrm{f}}$ & $\begin{array}{c}T_{\mathrm{e}} \\
(\mathrm{K})\end{array}$ \\
\hline HIGHION & 4.10 & $2.5 \mathrm{e} 18$ & $3.2 \mathrm{e} 36$ & 0.040 & $2.94 \mathrm{e} 06$ \\
MEDION & 3.37 & $1.5 \mathrm{e} 19$ & $6.4 \mathrm{e} 37$ & 0.023 & $1.44 / 1.22$ \\
LOWION & 3.60 & $2.5 \mathrm{e} 19$ & $3.6 \mathrm{e} 37$ & 0.004 & $10.2 / 3.54$ \\
\hline
\end{tabular}

${ }^{a}$ Assuming $\Delta r / r<1$, see the text.

${ }^{b}$ Predicted FM values are given for initial/final zone.

respectively. This constraint can be reformatted in terms of $U$ and $N_{\mathrm{H}}$ such that

$r \leq Q /\left(4 \pi c N_{\mathrm{H}} U\right)$

and

$n_{\mathrm{H}} \geq\left(4 \pi c N_{\mathrm{H}}^{2} U\right) / Q$.

Using the values of $U$ and $N_{\mathrm{H}}$ derived from the XSPEC fitting, we computed values for $r$ and $n_{\mathrm{H}}$ for each component which are listed in Table 3. We then generated new CLOUDY models for each component. In Table 3 , we list the predicted average electron temperatures, $T_{\mathrm{e}}$, and force multiplers (FM), the ratio of the total radiation cross-section to the Thomson cross-section, at the ionized face and final zones, for each model component. ${ }^{3}$

The emitting area $(A)$ of each component is computed from the ratio of the emission line and RRC luminosities, using the measured fluxes in Table 1 and assuming a distance of $15 \mathrm{Mpc}$, to the CLOUDY-predicted emission line and RRC fluxes. Specifically, $A=$ luminosity/flux. Additionally, we require the values of $A$ to be proportional to the relative normalizations of each component. Since the normalizations scale to the emission line fluxes, we rescaled them based on the re-computed values of $n_{\mathrm{H}}$. And, since the values of $N_{\mathrm{H}}$ are fixed, the fluxes and the normalizations scale as $n_{\mathrm{H}}$. Keeping the normalization of HIGHION fixed, those of MEDION and LOWION were increased by factors of 20.1 and 11.5,

\footnotetext{
${ }^{3}$ The 'ionized face' refers to the initial CLOUDY model zone, hence the part of a slab closest to the ionizing source. The 'final zone' is the last zone computed by CLOUDY in the determination of the radiative transfer through the gas.
}

respectively. The covering fraction of each component is calculated as $C_{\mathrm{f}}=A /\left(4 \pi r^{2}\right)$. Das et al. (2005) calculated a half-opening angle of the optical emission line bicone in NGC 4151 of $33^{\circ}$. If the X-ray emission line gas is confined to the bicone, $C_{\mathrm{f}} \leq 0.3$. Our predicted $C_{\mathrm{f}}$ are consistent with this scenario. The final values of $A$ and $C_{\mathrm{f}}$ for each components are listed in Table 3.

\subsubsection{Comparison to emission line measurements}

The model-predicted fluxes and luminosities of the emission lines and RRCs are compared with the measured values in Table 4. The last column of the table is the data/model ratio. Most of the features are well fitted, with $0.56<$ data/model $<1.74$, with the exception of the Si XIII triplet, as noted above. The contributions of the individual components are clearly distinguished, with emission from ions with IP, $>1.2 \mathrm{keV}$, e.g. Si XIV, Si XIII, and Mg XII, coming from HIGHION, those with the lowest IP, e.g. O VII $(138.08 \mathrm{eV})$, coming from LOWION, and MEDION contributing to all ionization states.

As noted in Section 2.2.1, we were unable to obtain accurate constraints on the widths of the RRCs. However, we were able to determine if the predicted electron temperatures $\left(T_{\mathrm{e}}\right)$ were consistent with the data. To do so, we refit the O VII and O VIII RRCs with a fixed ratio of the contributions from each model component (see Table 4) and fixed $k T_{\mathrm{e}}$, where $k$ is Boltzmann's constant. For O VII, assuming 90 percent at $T_{\mathrm{e}}=1.7 \times 10^{5} \mathrm{~K}$, from MEDION, and 10 percent at $T_{\mathrm{e}}=1.9 \times 10^{4} \mathrm{~K}$, from LOWION, this yielded a normalization $8.41 \mathrm{e}-05$ with 90 percent confidence range from $5.99 \mathrm{e}-05$ to $1.11 \mathrm{e}-04$. For O VIII, assuming 67 percent at $T_{\mathrm{e}}=$ $2.94 \times 10^{6} \mathrm{~K}$, from HIGHION, and 33 percent from MEDION, this yields a normalization of $1.33 \mathrm{e}-04$ with 90 per cent confidence 
Table 4. Comparison of measured and predicted emission line luminosities. ${ }^{a}$

\begin{tabular}{lcccccc}
\hline Line & Measured luminosity & HIGHION & MEDION & LOWION & Total $^{b}$ & Data/model \\
\hline Si XIV $\alpha$ & $7.40 \pm 0.74$ & 4.49 & 0.84 & & 5.33 & 1.39 \\
Si XIII r & $3.74 \pm 0.48$ & 1.55 & 2.21 & & 3.77 & 0.99 \\
Si XIII i & $1.79 \pm 0.28$ & 0.18 & 0.95 & & 1.13 & 1.59 \\
Si XIII f & $5.50 \pm 0.95$ & 0.41 & 2.00 & & 2.40 & 2.29 \\
Mg XII $\beta$ & $2.96 \pm 0.45$ & 1.04 & 0.67 & & 1.70 & 1.74 \\
Mg XII $\alpha$ & $4.83 \pm 0.52$ & 3.29 & 2.32 & & 5.61 & 0.86 \\
Ne X RRC & $6.06 \pm 1.35$ & 3.34 & 2.33 & & 5.67 & 1.07 \\
Mg XI r & $3.70 \pm 0.55$ & 0.56 & 3.44 & 0.07 & 4.07 & 0.91 \\
Mg XI i & $1.53 \pm 0.44$ & 0.04 & 2.25 & & 2.29 & 0.67 \\
Mg XI f & $4.73 \pm 0.71$ & 0.12 & 6.03 & 0.02 & 6.17 & 0.77 \\
Ne IX RRC & $2.46 \pm 0.44$ & 0.07 & 2.91 & 0.08 & 3.07 & 0.80 \\
Ne X $\alpha$ & $10.1 \pm 1.03$ & 4.07 & 9.36 & 0.12 & 13.5 & 0.75 \\
Ne IX r & $7.87 \pm 1.87$ & 0.41 & 6.10 & 0.45 & 6.96 & 1.13 \\
Ne IX i & $7.82 \pm 2.03$ & 0.03 & 5.93 & 0.22 & 6.18 & 1.27 \\
Ne IX f & $18.7 \pm 1.87$ & 0.09 & 18.5 & 0.75 & 19.3 & 0.97 \\
O VIII RRC & $13.5 \pm 1.93$ & 8.92 & 14.9 & 0.27 & 24.1 & 0.56 \\
O VII RRC & $15.3 \pm 3.55$ & 0.05 & 8.44 & 1.03 & 9.52 & 1.61 \\
O VIII $\alpha$ & $38.4 \pm 7.03$ & 7.86 & 35.2 & 1.87 & 44.9 & 0.86 \\
O VII r & $9.62 \pm 5.72$ & 0.44 & 9.36 & 2.47 & 12.3 & 0.78 \\
O VII f & $78.4 \pm 17.6$ & 0.12 & 47.0 & 17.5 & 64.6 & 1.21 \\
\hline${ }^{a}$ In units of $10^{38}$ erg s ${ }^{-1}$. & \multicolumn{5}{c}{ Th determine the line luminosities, the predicted line fluxes were multiplied by the emitting areas listed in Table 3. }
\end{tabular}

range $1.02 \mathrm{e}-04$ to $1.65 \mathrm{e}-04$. Hence, the model predictions are consistent with the data, but the values are not well constrained. However, there is no evidence that the values are significantly higher, especially for $\mathrm{O}$ vII, hence there is no need for a collisionally ionized component in the spectral fitting.

With the large column densities required by the fit to the HETG spectra, the models also predict significant UV line emission, the strongest of lines being OVI $\lambda \lambda 1032,1038$. The combined $\mathrm{O}$ VI luminosity from the models is $L_{\mathrm{OVI}}=1.9 \times 10^{40} \mathrm{erg} \mathrm{s}^{-1}$, $\sim 85$ percent of which is from LOWION. Based on FUSE spectra, the OVI flux from the NLR is $2 \times 10^{-12} \mathrm{erg} \mathrm{cm}^{-2}$ $\mathrm{s}^{-1}$, or $L_{\mathrm{OVI}}=5.4 \times 10^{40} \mathrm{erg} \mathrm{s}^{-1}$ (Armentrout et al. 2007). Therefore, while the X-ray gas is predicted to have a strong UV signature, the models are consistent with the UV emission line constraints.

\subsubsection{Masses and mass outflow rates}

The mass of emission line gas for each component is $M=\mu m_{\mathrm{p}} A N_{\mathrm{H}}$, where, $m_{\mathrm{p}}$ is the mass of a proton, and $\mu=1.4$, which accounts for the mass of helium and the heavy elements, at roughly solar abundances. This may also be written as $M=4 \pi r^{2} C_{\mathrm{f}} \mu m_{\mathrm{p}} n_{\mathrm{H}}$ $\Delta r$, where $n_{\mathrm{H}} \Delta r=N_{\mathrm{H}}$. For the three model components, we obtain the following: HIGHION, $118 \mathrm{M}_{\odot}$; MEDION, $2.59 \times 10^{3}$ $\mathrm{M}_{\odot}$; and LOWION, $4.17 \times 10^{3} \mathrm{M}_{\odot}$. The mass outflow rate is $\dot{M}_{\text {out }}=4 \pi r N_{\mathrm{H}} \mu m_{\mathrm{p}} C_{\mathrm{f}} v_{\mathrm{r}}$. Assuming the average value of $v_{\mathrm{r}}$ given in Section 2.2.2, we obtain the following: HIGHION, $0.03 \mathrm{M}_{\odot} \mathrm{yr}^{-1}$; MEDION, $0.11 \mathrm{M}_{\odot} \mathrm{yr}^{-1}$; and LOWION, 0.09 $\mathrm{M}_{\odot} \mathrm{yr}^{-1}$. Although not strictly lower limits, these values were computed assuming the components were at distances computed by requiring $\Delta r / r<1$, and we did not use a deprojected $v_{\mathrm{r}}$. The summed mass outflow rate is $0.23 \mathrm{M}_{\odot} \mathrm{yr}^{-1}$, which is close to the optical/ $\mathrm{UV}^{4}$ rate from within $12 \mathrm{pc}$ of the nucleus, $\approx 0.3 \mathrm{M}_{\odot} \mathrm{yr}^{-1}$ computed by Crenshaw et al.

\footnotetext{
${ }^{4}$ The term Optical/UV refers to the values derived from the analysis of STIS spectra covering its full UV to near-IR bandpass.
}

(2015). However, given that several of the emission lines extend more than $100 \mathrm{pc}$ from the nucleus (see Fig. 2) and the deprojected velocities are likely higher than $v_{\mathrm{r}}$ (Crenshaw et al. 2015), the values of $M$ and $\dot{M}_{\text {out }}$ can be significantly greater. We address this in the next section.

\section{ANALYSIS OF EXTENDED EMISSION}

\subsection{Zeroth-order line profiles}

The soft X-ray emission is resolved in NGC 4151 (Ogle et al. 2000) and extends beyond $1 \mathrm{kpc}$ (Wang et al. 2011a). As discussed in Section 2.1, the zeroth-order image shows $\mathrm{H}$ - and He-like O and $\mathrm{Ne}$ lines extending to $\geq 100$ pcs from the nucleus. For the extended gas to be in a sufficiently high state of ionization to produce these lines, the densities must decrease with distance, as suggested by Wang et al. (2011b). Line emissivities are proportional to $n_{\mathrm{H}}^{2}$ (Osterbrock \& Ferland 2006). Therefore, even though the line fluxes decrease with distance, there may be a significant amount of mass associated with the extended emission.

While in NGC 1068, there are enough counts in the \pm first-order HETG spectra to obtain accurate emission line fluxes outside the inner nucleus (see Kallman et al. 2014), this is not the case for NGC 4151. Hence, we used the zeroth-order spectral images to measure the extended emission. We measured the fluxes in regions of $0.5 \mathrm{arcsec}$ in length and $3.0 \mathrm{arcsec}$ in width, centred at the nucleus, along a position angle of $140^{\circ}$. Our extraction window is shown, superimposed on an archival HST/WFPC2 [O III] image, in the left-hand panel of Fig. 8. The right-hand panel of Fig. 8 shows the $[\mathrm{O}$ III] emission profile over the same window. In Fig. 9, we show the extracted Ne IX rif and Ne X $\alpha$ profiles, compared with the 2.05-2.25 keV continuum, which was chosen because it is free of strong line emission. The emission line profiles are clearly broader than the continuum and show structure that is similar to the [O III] profile.

We determined the fraction of emission in each 0.5 arcsec bin by dividing counts in the bin by the total counts for each line. These 

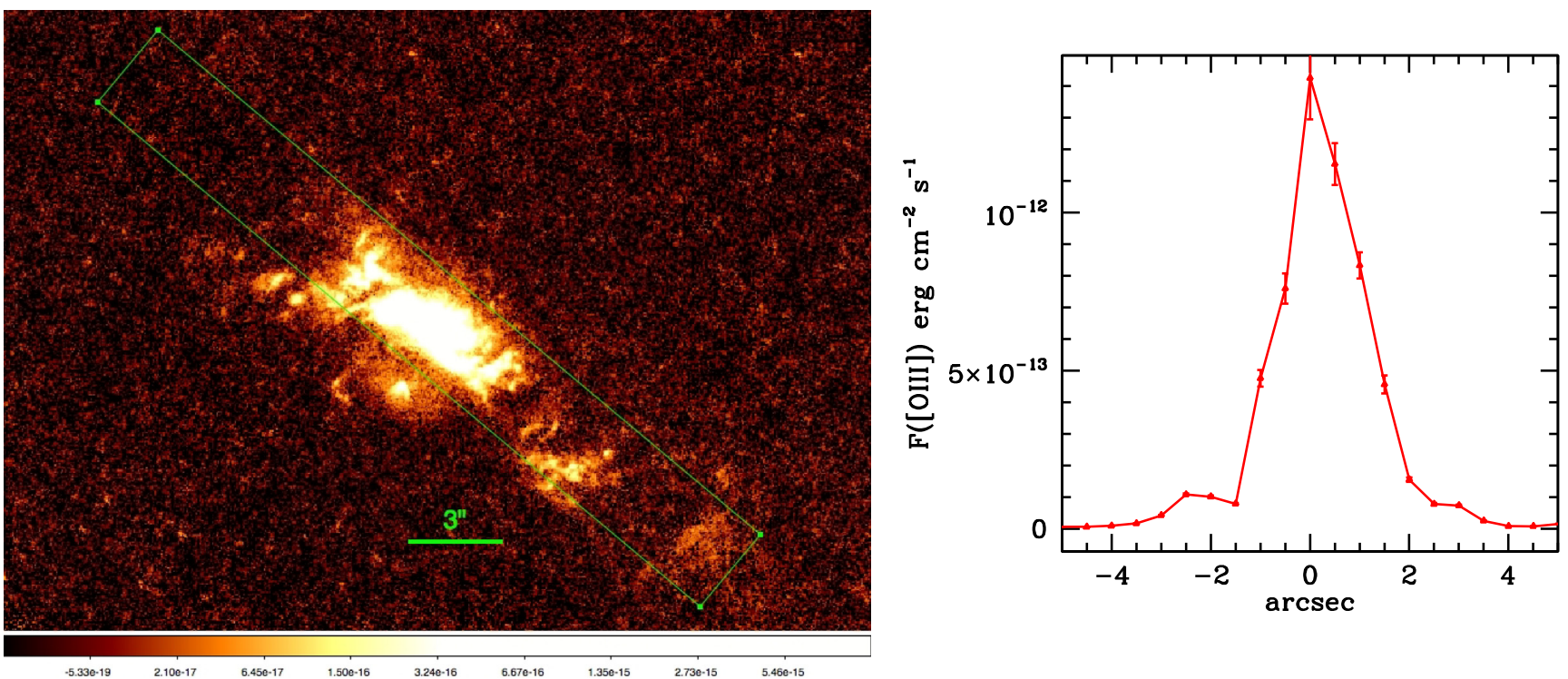

Figure 8. Left-hand panel: The $0.5 \operatorname{arcsec} \times 3.0$ arcsec extraction region used to obtain the Ne IX and Ne X profiles, along position angle $140^{\circ}$, superimposed upon an archival WFPC2 [O III] image of NGC 4151. North up, east to left. Right-hand panel: [O III] fluxes measured within the same extraction bins; the error bars show the standard deviations which were calculated by measuring the root mean squares of fluxes in the extraction window and multiplying this number by the square root of the number of pixels in this extraction region. Positive positions are those south-west (SW) of the nucleus.
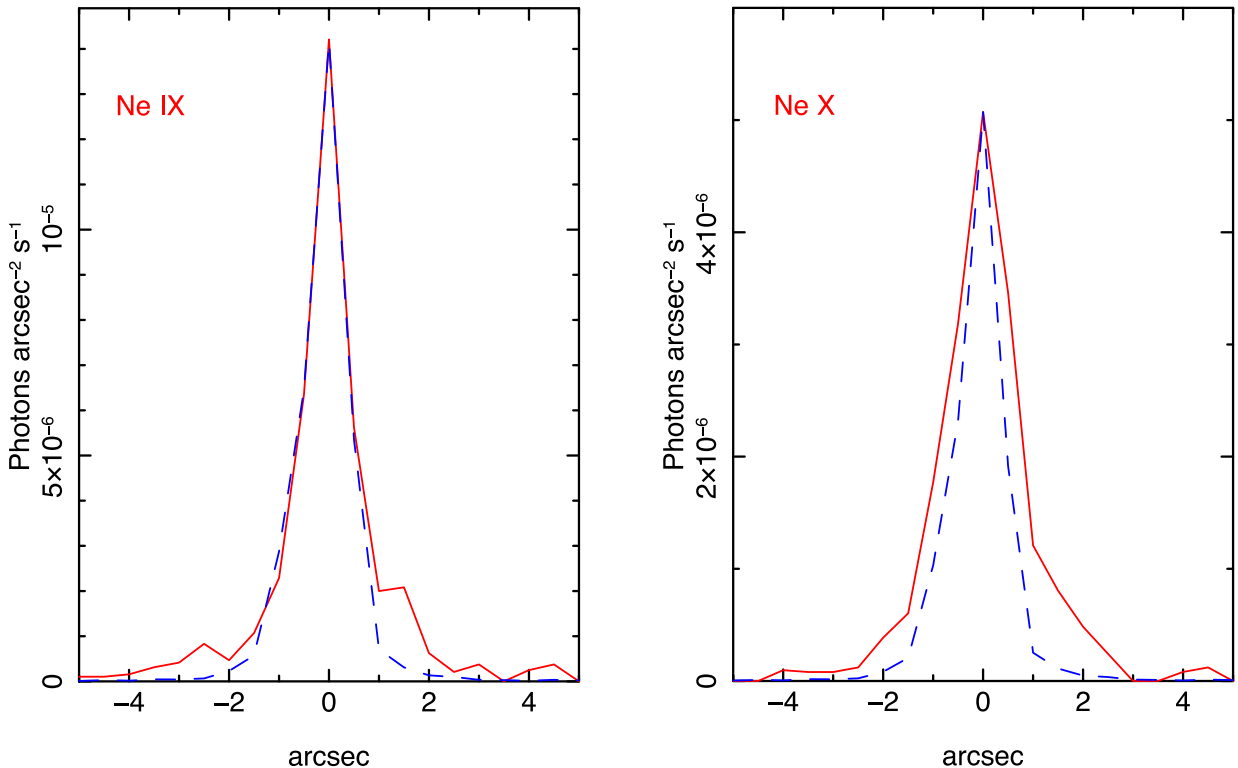

Figure 9. Left-hand panel: Ne IX emission line profile (in red) as a function of position. The fluxes were measured from the zeroth-order HETG spectrum. The blue dashed line is the continuum; positive positions are SW of the nucleus. Right-hand panel: Ne X emission line profile.

were converted to line luminosities as a function of distance by multiplying by the total luminosity of the lines, listed in Table 4 . In our analysis, we only considered bins for which the signal-to-noise ratios were $>2$.

\subsection{Estimating the mass of the extended gas}

In order to fit the spatial resolved emission line profiles, we generated CLOUDY models for distances corresponding to the centre points of the 0.5 arcsec bins. The distances from the ionizing source for each model were determined assuming an inclination angle of $45^{\circ}$, which is the inclination of the bicone axis with respect to the plane of the sky (Das et al. 2005). Because we are mapping $\mathrm{Ne}$ IX rif and $\mathrm{Ne} \mathrm{X} \alpha$, we only ran these models for the HIGHION and MEDION. ${ }^{5}$ In doing so, we assumed that density decreases as $n_{\mathrm{H}} \propto r^{-1.65}$, which is the same as for the optical/UV emission line gas used in Crenshaw et al. (2015). Crenshaw et al. (2015) based

${ }^{5}$ We chose to map the $\mathrm{Ne} \mathrm{X}$ and $\mathrm{Ne}$ IX emission, rather than O VIII and $\mathrm{O}$ VII, because the latter are at lower energies, where the decrease in ACIS sensitivity is more severe, and are strongly dependent on the predictions from the LOWION, which is not well constrained. 
Table 5. CLOUDY model parameters for spatially resolved Ne IX and Ne X emission.

\begin{tabular}{lcrrrc}
\hline Model & distance & $\log U$ & $\begin{array}{c}\log \left(n_{\mathrm{H}}\right)^{a} \\
\left(\mathrm{~cm}^{-3}\right)\end{array}$ & $\begin{array}{c}\log \left(N_{\mathrm{H}}\right)^{b} \\
\left(\mathrm{~cm}^{-2}\right)\end{array}$ & $\mathrm{FM}^{c}$ \\
\hline HIGHION & $12 \mathrm{pc}$ & 0.59 & 2.17 & 21.74 & $2.74 / 1.95$ \\
& $50 \mathrm{pc}$ & 0.37 & 1.15 & 21.34 & $4.86 / 3.26$ \\
& $100 \mathrm{pc}$ & 0.26 & 0.66 & 20.85 & $6.68 / 5.37$ \\
& $153 \mathrm{pc}$ & 0.20 & 0.35 & 20.54 & $10.0 / 7.22$ \\
& $205 \mathrm{pc}$ & 0.16 & 0.14 & 20.33 & $11.9 / 8.78$ \\
& $257 \mathrm{pc}$ & 0.12 & -0.02 & 20.17 & $45.0 / 17.2$ \\
& $309 \mathrm{pc}$ & 0.09 & -0.15 & 20.04 & $48.2 / 19.1$ \\
& $360 \mathrm{pc}$ & 0.07 & -0.26 & 19.93 & $50.4 / 20.8$ \\
& $412 \mathrm{pc}$ & 0.05 & -0.36 & 19.83 & $52.7 / 22.6$ \\
& $464 \mathrm{pc}$ & 0.03 & -0.44 & 19.67 & $18.9 / 14.9$ \\
& $12 \mathrm{pc}$ & 0.05 & 2.71 & 22.28 & $16.7 / 4.44$ \\
& $50 \mathrm{pc}$ & -0.17 & 1.69 & 21.88 & $26.0 / 6.92$ \\
& $100 \mathrm{pc}$ & -0.27 & 1.19 & 21.38 & $31.8 / 9.96$ \\
& $153 \mathrm{pc}$ & -0.34 & 0.89 & 21.06 & $37.4 / 12.7$ \\
& $205 \mathrm{pc}$ & -0.38 & 0.68 & 20.81 & $41.1 / 14.9$ \\
& $257 \mathrm{pc}$ & -0.42 & 0.52 & 20.71 & $45.0 / 17.2$ \\
& $309 \mathrm{pc}$ & -0.45 & 0.39 & 20.58 & $48.2 / 19.1$ \\
& $360 \mathrm{pc}$ & -0.47 & 0.28 & 20.27 & $50.4 / 20.8$ \\
& $412 \mathrm{pc}$ & -0.49 & 0.18 & 20.37 & $52.7 / 22.6$ \\
& $464 \mathrm{pc}$ & -0.50 & 0.09 & 20.28 & $53.9 / 24.0$ \\
\hline
\end{tabular}

${ }^{a}$ Based on density law $n_{\mathrm{H}} \propto r^{-1.65}$.

${ }^{b}$ Assuming $\Delta r / r<1$ or $\Delta r$ fixed at $50 \mathrm{pc}$; for a detailed explanation see Section 4.2.

${ }^{c}$ Predicted FM values are given for initial/final zone.

${ }^{d}$ The drop in FM is due to the small value for $N_{\mathrm{H}}$, which results in a higher average ionization compared to the model predictions for $412 \mathrm{pc}$. CLOUDY calculates the radiation transfer and physical conditions over zones of finite size. Typically these are scaled in units of optical depth, rather than physical depth. So, if the zone is small in depth, the average ionization can be higher than a similar zone that penetrates deeper into the gas. Since the depth of the model at $412 \mathrm{pc}$ is small compared to the other regions, it is of higher average ionization. Hence, the FM, which depends on the ionization state of the gas, is lower than it would be for a model with the same $U$, but with a greater $N_{\mathrm{H}}$.

their density law on our analysis of HST/STIS spectra (Kraemer et al. 2000), in which we used density diagnostics, such as the [S II] $6716 \AA / 6731$ Å ratio, and constraints from photoionization models. The density laws for each component were normalized using the densities obtained for the global models (Table 3). Although there is no direct evidence for such a density law for X-ray gas, there are two reasons why it is plausible. First, the fact that the gas is being mapped out by Ne IX and Ne X suggests that the ionization state of the gas is not dropping precipitously, which implies that the density could fall as $r^{-2}$, as proposed by Wang et al. (2011b). Secondly, as we will discuss in the next section, the X-ray gas may arise from expansion of the optical emission line gas, which could lead to the same density law for both. We constrained $N_{\mathrm{H}}$ by requiring $\Delta r / r<1$, as before, although for radial distances $>25 \mathrm{pc}, r$ is the deprojected size of the extraction bin. Hence, $N_{\mathrm{H}}$ decreases with $r$ as $n_{\mathrm{H}}$ is decreasing. The CLOUDY model parameters are listed in Table 5.

In order to calculate the masses in each bin, we used the line luminosities and the CLOUDY-predicted fluxes for Ne IX rif and $\mathrm{Ne} \mathrm{X} \alpha$ to solve for the emitting areas of each component, $A_{\mathrm{MED}}$ and $A_{\mathrm{HIGH}}$, using the following equations:

$F(\mathrm{Ne} \mathrm{IX})_{\mathrm{MED}} \times A_{\mathrm{MED}}+F(\mathrm{Ne} \text { IX })_{\mathrm{HIGH}} \times A_{\mathrm{HIGH}}$

$=L_{\mathrm{Ne} \text { IX }} \times f r a c 9$ and,

$$
\begin{aligned}
& F(\mathrm{NeX})_{\mathrm{MED}} \times A_{\mathrm{MED}}+F(\mathrm{NeX})_{\mathrm{HIGH}} \times A_{\mathrm{HIGH}} \\
& \quad=L_{\mathrm{Ne}} \times \text { frac } 10
\end{aligned}
$$

where $F(\mathrm{Ne} \text { IX })_{\mathrm{MED}}, F(\mathrm{~N} e \text { IX })_{\mathrm{HIGH}}, F(\mathrm{~N} e \mathrm{X})_{\mathrm{MED}}$, and $F(\mathrm{~N} e \mathrm{X})_{\mathrm{HIGH}}$ are the CLOUDY-predicted fluxes from each component, frac 9 and frac 10 are the fractions of the emission line fluxes in each bin, and $L_{\mathrm{Ne} \text { IX }}$ and $L_{\mathrm{Nex}}$ are the line luminosities (see Table 4).

In order to check that the set of models for the extended emission still provided a good fit to the spectra, we used the values for $A_{\mathrm{MED}}$ and $A_{\mathrm{HIGH}}$ to compute the luminosities of other emission lines. The predictions for the Mg XI $r$ and $f$ and Si XIII $r$ lines remained well within the measurement uncertainties. The fit for $\operatorname{Si} \operatorname{XIII} f$ worsened (data/model $=5.3$ ), but the line was not well fit by the initial model (see Section 3.2.2). However, the fit for Mg XII $\alpha$ was now poor $($ data/model $=2.3$ ), and the new models predicted only 15 per cent of the Si XIV $\alpha$. Nevertheless, these results are consistent with the unresolved profiles for these lines (see Fig. 2). In summary, while the models provide a good fit to the resolved emission lines, there is still need for a component of high-ionization gas close to the AGN.

Once we determined the $A$ 's for each component, we calculated $M$ for each bin using the model $N_{\mathrm{H}}$ (Table 5), as described in Section 3.2.3. The final values are plotted in the left-hand panel of Fig. 10. The low value for $M$ in the centre bin is due to the high density (see Table 5). The reason for this is that the emissivity of the gas goes as $n_{\mathrm{H}}^{2}$, hence, less mass can produce the same emission. The uncertainties in the $\mathrm{X}$-ray masses are due to photon-counting statistics, while those in position are due to the size of the extraction bins. The line emissivities are proportional, hence the values of $A$ are inversely proportional, to $n_{\mathrm{H}}$ (see Osterbrock \& Ferland 2006. The total mass of the ionized gas is $5.4( \pm 1.1) \times 10^{5} \mathrm{M}_{\odot}$.

As shown in Crenshaw et al. (2015) and Revalski et al. (2018b), the optical outflows in NGC 4151 extend out to a distance of $\sim 150$ pc. To compare the mass distribution of the X-ray emission line gas to that of the optical/UV gas within the outflow region, we summed the X-ray masses from the same distances to the NE and $\mathrm{SW}$ of the nucleus. We summed the masses from optical analysis to correspond to the bin sizes used for the X-ray extraction; the errors in the mass values are those from Crenshaw et al. (2015), added in quadrature to account for the binning. The results are shown in the right-hand panel of Fig. 10. The totals are roughly similar. Note that the drop in optical/UV mass in most distant zone is primarily an artefact, resulting from the truncation of the optical measurement at $132 \mathrm{pc}$. The total mass of $\mathrm{X}$-ray gas within the outflow regions is $3.9( \pm 0.4) \times 10^{5} \mathrm{M}_{\odot}$, compared to $\approx 3 \times 10^{5} \mathrm{M}_{\odot}$ for the optical/UV component.

\subsection{Mass outflow rates in the extended gas}

Using the derived masses for the $\mathrm{X}$-ray gas, we calculated the values of $\dot{M}_{\text {out }}$ as a function of distance, as described in Section 3.2.3. However, since the physical depth, or $\Delta r$, of each model component is now limited by the size of the extraction bins, rather than requiring $\Delta r / r<1$, the equation for the mass outflow rate can be rewritten as $\dot{M}_{\text {out }}=\frac{M v_{\mathrm{r}}}{\Delta r}$ (e.g. Revalski et al. 2018a). In computing $\dot{M}_{\text {out }}$, we assumed that the X-ray gas exhibited the same kinematics as the [O III] gas. There are two main reasons as to why this is a plausible assumption. First, as noted in Section 2.2.2, while the average velocity centroid for the X-ray lines is higher than that of the collapsed [O III] centroid, this is likely due to absorption of the 

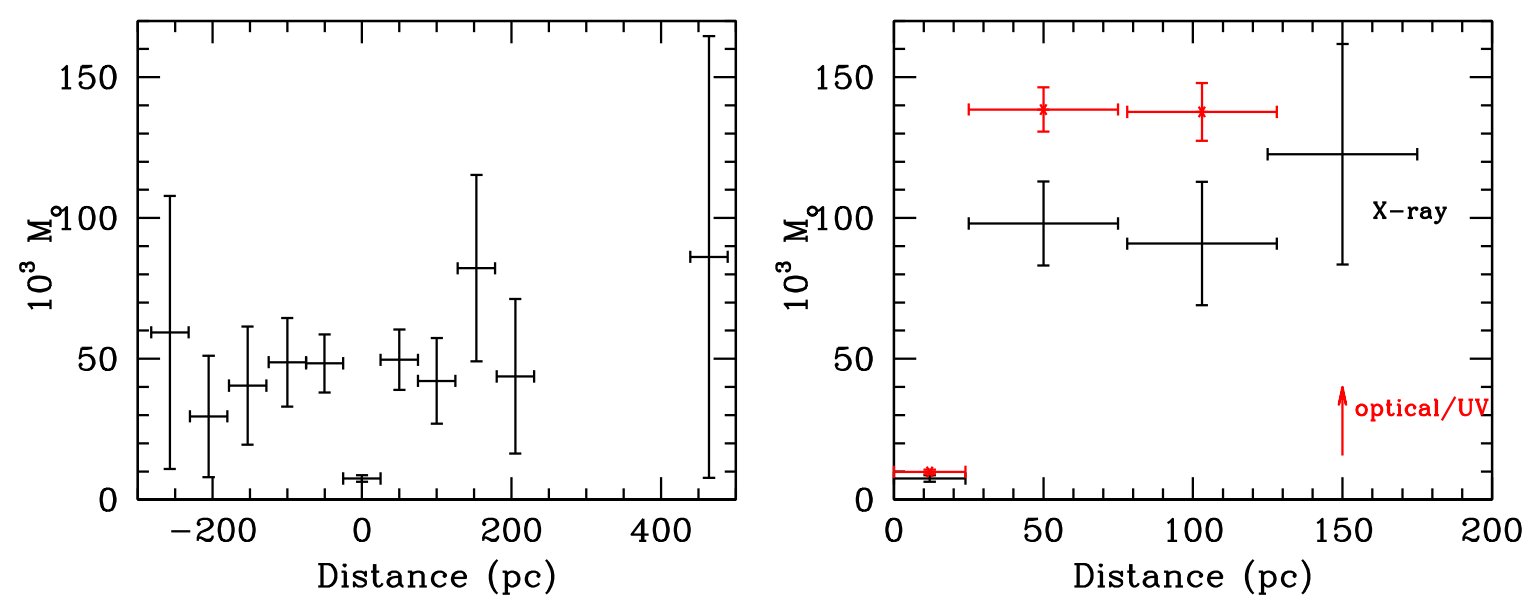

Figure 10. Left-hand panel: Computed values of $M$, in units of $10^{3}$ solar masses, as a function of deprojected distance, in parsecs. The positive/negative distances correspond to point SW/NE of the nucleus, respectively. Uncertainties in $M$ value reflect photon counting statistics, while the uncertainties in the distances correspond to the bin sizes. Right-hand panel: Computed $M$ values at the same radial distances (SW and NE values summed) for the HETG analysis (black crosses), compared to those from the STIS optical/UV analysis (red asterisks), from Crenshaw et al. (2015) and Revalski et al. (2018b). The optical/UV points are summed to correspond with the HETG extraction bin sizes. Uncertainties in the optical/UV points are those from Crenshaw et al., and have been added in quadrature to account for the binning.

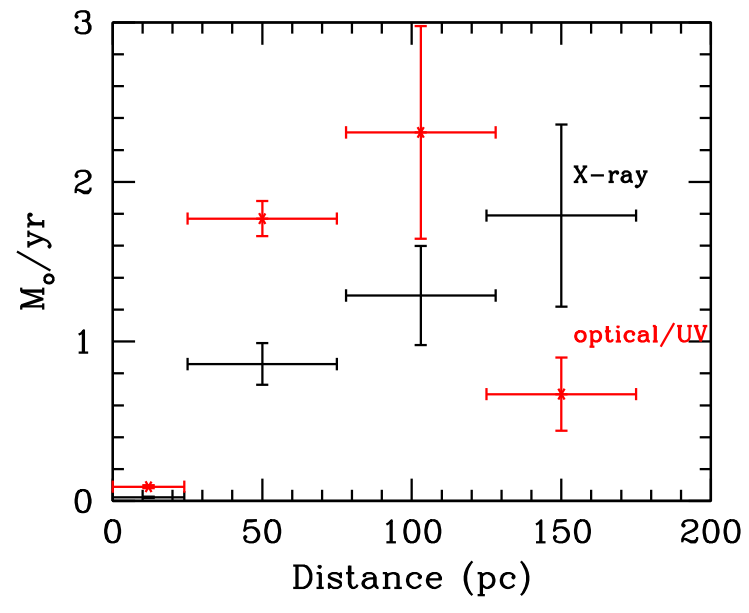

Figure 11. Computed values of $\dot{M}_{\text {out }}$ as a function of distance, from the HETG analysis (black crosses), compared to those from the STIS optical/UV analysis (red asterisks). Both data sets, the $\dot{M}_{\text {out }}$ values were computed using flux-weighted velocities within each bin from the STIS analysis. Uncertainties include both the $M$ values and the flux-weighted velocities.

soft X-ray emission NE of the nucleus. Secondly, as shown in the Figs 8 and 9, the profiles of the [O III], Ne IX, and NeX lines are similar when measured over the same extraction bins.

In Fig. 11, we show $\dot{M}_{\text {out }}$ as a function of deprojected radial distance, for both the X-ray and optical/UV gas. The errors plotted for the X-ray $\dot{M}_{\text {out }}$ 's, in addition to the contribution from the mass estimates, include those of the [O III] velocity measurements. The errors for the optical/UV points are from Crenshaw et al. (2015), again added in quadrature to account for the binning. The X-ray $\dot{M}_{\text {out }}$ 's are similar to those in the optical/UV gas, although the former does not drop off at the highest distance. Note that the smaller size for the final bin in the optical/UV data also enters into the equation for $\dot{M}_{\text {out }}$, in a manner that compensates for the smaller mass in that bin (see Section 3.2.3) hence the drop-off in $\dot{M}_{\text {out }}$ is not an artefact in this case. The maximum X-ray $\dot{M}_{\text {out }}=1.80 \pm 0.57 \mathrm{M}_{\odot} \mathrm{yr}^{-1}$, as compared to $2.31 \pm 0.67 \mathrm{M}_{\odot} \mathrm{yr}^{-1}$ for the optical/UV gas. Note the latter is somewhat smaller than the $\sim 3 \mathrm{M}_{\odot} \mathrm{yr}^{-1}$ given in Revalski et al. (2018b); this is due to the averaging of the velocity within the extraction bin. While the outflow rates are similar, the possibility of a greater extent for the X-ray outflows suggests that its importance in mass outflow, hence AGN feedback, may have not been fully appreciated, as we discuss in Section 5.

From these results, we determined a peak $L_{\mathrm{KE}}=2.92( \pm 1.22) \times$ $10^{41} \mathrm{erg} \mathrm{s}^{-1}$, at a distance of $150 \mathrm{pc}$. This is similar to the value derived by Wang et al. (2011b) and confirms that the power of the X-ray outflow is comparable to that of the optical/UV gas (Crenshaw et al. 2015). Adding in the optical/UV contribution, $L_{\mathrm{KE}}$ is $\sim 0.5$ per cent of $L_{\mathrm{bol}}$, which is at the limit for efficient feedback of $0.5-5$ per cent (Hopkins \& Elvis 2010), although these limits may not be relevant for such a sub-Eddington AGNs (King \& Pounds 2015).

\section{DISCUSSION}

As shown in Section 4, the extended X-ray emission line gas in NGC 4151 may possess similar mass, mass distributions, and mass outflow rates as the optical/UV gas. However, the low value of $L_{\text {edd }} / L_{\text {bol }}$ in NGC 4151 calls into question the physical process by which the gas is accelerated. For example, the possibility of radiative acceleration of the gas can be determined by comparing the predicted $F M$ s (see Table 3 ) to $L_{\text {edd }} / L_{\text {bol }}$. Specifically, radiative acceleration is proportional to $F M \times L_{\text {bol }}$ (e.g. Das, Crenshaw \& Kraemer 2007). For $F M=1$, the only source of opacity is electron scattering, via the Thomson cross-section, and radiative acceleration can only occur if the source is radiating at $L_{\text {edd }}$. At lower ionization, the opacity and, hence, $F M$ increases. Therefore, radiative acceleration can occur in sources with $L_{\mathrm{bol}}=L_{\mathrm{edd}} / F M$. Using our estimate for $L_{\mathrm{bol}}$, radiative acceleration requires $F M \geq 25$. Therefore, radiative acceleration is not possible for HIGHION, while it would marginally possible for MEDION if $L_{\text {bol }}$ is closer to the value calculated by Wang et al. (2011b). LOWION dynamics are consistent with radiative acceleration, but the large difference in FM across the zone suggests that LOWION cannot be dynamically stable (and its physical characteristics are not well constrained by our models).

An alternative means of acceleration for highly ionized gas, in sub-Eddington AGNs, is via magneto hydrodynamic (MHD) 
processes. In fact, MHD has been suggested as the acceleration mechanism for the most highly ionized absorbers in NGC 4151 (Kraemer et al. 2005; Kraemer, Tombesi \& Bottorff 2018). Effective MHD requires strong magnetic fields, such as those associated with the accretion disc (Blandford \& Payne 1982), which infers that the outflows originate close to the AGN. However, Crenshaw et al. (2015) argued that there was significantly more optical/UV gas in the NLR of NGC 4151 than could be supplied by outflows from the inner nucleus. This is almost certainly the case for the X-ray gas, given the similar masses and mass outflow rates to the optical/UV component. Instead, they suggested that most of the gas has been accelerated in situ, and originated in dust spirals that had crossed the illumination cone. This scenario has been explored in more detail by Fischer et al. (2017), who demonstrated that outflows in the NLR of the Seyfert 2 Mrk 573 could be explained by in situ acceleration of material in the host galaxy's disc. As such, it is unlikely that the $\mathrm{X}$-ray gas is part of an MHD flow.

If the bulk of the extended X-ray gas originates in the host galaxy's disc, and was accelerated in situ, the FM's listed in Table 5 are more relevant for evaluating the efficacy of radiative acceleration. However, at these larger radial distances, the enclosed stellar mass becomes a dominant source of gravitational deceleration, as shown in Das et al. (2007). Based on their results, an $F M \sim 50$ is far too low to launch an outflow in NGC 1068, which is more luminous than NGC 4151 (see Kraemer et al. 2015). Therefore, radiative acceleration would be unlikely even if the outflows were launched at a time when NGC 4151 was radiating at closer to its Eddington limit.

Embedded dust may play an important role in AGN-driven winds (e.g. Ishibashi, Fabian \& Maiolino 2018). Specifically, dust grains may provide sufficient opacity for radiative acceleration of highly ionized winds in sub-Eddington AGNs. To test this, we generated models for the MEDION component which included a Galactic interstellar medium dust fraction, using the 'ISM' option (Ferland et al. 2017). Under these conditions, the CLOUDY models predict $F M \sim 300$. Using the expression for radial velocity as a function of distance given in Das et al. (2007) and their enclosed mass profile, ${ }^{6}$ we determined that dusty winds cannot be effectively launched at distances $\gtrsim 10 \mathrm{pc}$. Hence, while dust may play an important role in the acceleration of highly ionized close to the AGN, the associated increase in $F M$ is not sufficient to account for the generation of winds further into the host galaxy.

Another possibility is that the X-ray gas was in a lower state of ionization, by virtue of being higher density, when initially accelerated. Indeed, this has been suggested for the radiative acceleration of Ultra Fast Outflows in AGNs (Hagino et al. 2015). One scenario is that the X-ray gas arises as the optical/UV gas expands. Photoionized gas in which [O III] is a dominant coolant would have $T_{\mathrm{e}} \sim 15000 \mathrm{~K}$ (Osterbrock \& Ferland 2006), which results in a sound speed $\sim 2 \times 10^{6} \mathrm{~cm} \mathrm{~s}^{-1}$. Unless somehow confined, the gas would become highly rarefied close to its point of origin. Based on the results from Mrk 573 (Fischer et al. 2017), it does appear that knots of [O III] emission can travel $10 \mathrm{~s}$ of pcs, which suggests some confinement, but the lack of high-velocity gas beyond several 100 pcs from the AGN is evidence that these knots eventually expand to the point that they are too highly ionized to be

\footnotetext{
${ }^{6}$ Das et al. (2007) used the mass profile for NGC 1068; since NGC 4151 has been classified as an earlier type spiral galaxy than NGC 1068 (de Vaucouleurs et al. 1991), it is likely that the enclosed mass at the same radial distance is greater.
}

detected in [O III]. Therefore, it is plausible that the X-ray outflow arises from optical/UV gas that is accelerated in situ.

\section{CONCLUSIONS AND SUMMARY}

We have analysed Chandra/HETG spectra of NGC 4151. The data were taken with an orientation to ensure that the axis of the NLR bicone was perpendicular to the dispersion direction, which enables us to map the extended emission line structure. Our main conclusions are as follows.

(1) In the zeroth-order spectral image, the continuum and lines with energies $>1.3 \mathrm{keV}$ are compact and centred on the nucleus. The $\mathrm{Si} \mathrm{K} \alpha$ and $\mathrm{Fe} \mathrm{K} \alpha$ lines are also strongly centrally peaked, consistent with an origin in the putative torus. On the other hand, the Ne X, Ne IX, O VIII, and O VII lines are all clearly extended along a NE-SW direction, out to an undeprojected distance of $>200 \mathrm{pc}$. This is in agreement with previous Chandra studies (e.g. Ogle et al. 2000; Wang et al. 2011a) and indicates that much of the soft X-ray emission arises in the NLR.

(2) The emission lines have an average radial velocity $v_{\mathrm{r}}=$ $-230_{-370}^{-90} \mathrm{~km} \mathrm{~s}^{-1}$, with no clear trend with IP. This is higher than the value obtained from the collapsed STIS spectra (Crenshaw et al. 2010), which we attribute to absorption of redshifted soft X-ray emission, NE of the nucleus, either by gas in the disc of the host galaxy or material associated with X-ray absorbers. Taking that into account, it is plausible that optical and X-ray outflows have similar kinematics.

(3) We were able to fit the first-order HETG spectra with a model consisting of a reflection component, and absorbed power law, with a small fraction of unabsorbed continuum, and three emission components. The absorption and emission components, or MTABLES and ATABLES, were generated with the photoionization code CLOUDY (Ferland et al. 2013, 2017). The models for the ATABLES were parametrized with $\log N_{\mathrm{H}} / \log U$ of $22.5 / 1.0$, 22.5/0.19, and 23.0, -0.50 , and named HIGHION, MEDION, and LOWION, respectively. HIGHION and MEDION are similar to the two highest ionization components used to fit the $X M M / \mathrm{RGS}$ spectra (Armentrout et al. 2007). On the other hand, LOWION, while required by the spectral fitting, is not well constrained, primarily due to the lack of sensitivity of Chandra at the lowest energies.

(4) As shown in Table 4, the emission line fluxes predicted by the CLOUDY models provide a good fit to the observed emission lines. Hence, we used the models as the basis for analysing the extended emission. To model the extent, we used the zeroth-order emission line profiles for Ne IX and Ne X (see Fig. 9) and assumed that the X-ray gas followed the same density law as the optical/UV gas (Crenshaw et al. 2015), or $n_{\mathrm{H}} \propto r^{-1.65}$. Masses were determined for each emission component within $50 \mathrm{pc}$ bins, to $r>200 \mathrm{pc}$. The summed masses are shown in Fig. 10. We derived a total mass of $5.4( \pm 1.1) \times 10^{5} \mathrm{M}_{\odot}$.

(5) By assuming the same velocity profile derived from the STIS analysis, we were able to use the models for the extended emission to compute $\dot{M}_{\text {out }}$ as a function of $r$. We obtain a maximum X-ray $\dot{M}_{\text {out }}=1.80 \pm 0.57 \mathrm{M}_{\odot} \mathrm{yr}^{-1}$, as compared to $2.31 \pm 0.67 \mathrm{M}_{\odot} \mathrm{yr}^{-1}$ for the optical/UV gas. Our value is close to that derived by Wang et al. (2011b) in their Chandra imaging analysis. Note, we did not include the contribution from LOWION, hence the true X-ray mass outflow rate may be somewhat higher.

(6) Although the kinematics of the X-ray gas appears to resemble the optical/UV gas, its relatively high-ionization state calls into question the efficiency of radiative acceleration. Although the 
presence of dust mitigates this, the corresponding increase in crosssection is not sufficient to overcome the gravity of the enclosed stellar mass, presuming, as we argue, most of the gas is launched in situ, in the plane of the host galaxy. One possible explanation is that the optical/UV gas is not fully confined. Hence, the X-ray component arises from thermal expansion of lower ionization, more efficiently accelerated gas.

In summary, based on our results, the X-ray emission line gas has a similar mass and peak mass outflow rate as the optical/UV gas. Therefore, it is an important component in AGN-driven winds. Furthermore, it does not appear to exhibit a drop in $\dot{M}_{\text {out }}$ for $r>$ $100 \mathrm{pc}$. That may indicate that the X-ray component has a greater effect on the host galaxy than the optical/UV gas and that X-ray winds might be a more efficient mechanism for AGN feedback. It would be interesting to see if there is a similar, or greater, role for $\mathrm{X}$-ray winds in higher luminosity AGNs.

\section{ACKNOWLEDGEMENTS}

Support for this work was provided by the National Aeronautics and Space Administration (NASA) through Chandra Award Number G04-15106X issued by the Chandra X-ray Observatory Center, which is operated by the Smithsonian Astrophysical Observatory for and on behalf of the National Aeronautics Space Administration under contract NAS8-03060, and from program GO-13508, support for which was provided by NASA through a grant from the Space Telescope Science Institute, which is operated by the Association of Universities for Research in Astronomy, Inc., under NASA contract NAS5-26555. This research has made use of data and/or software provided by the High Energy Astrophysics Science Archive Research Center (HEASARC), which is a service of the Astrophysics Science Division at NASA/Goddard Space Flight Center (GSFC) and the High Energy Astrophysics Division of the Smithsonian Astrophysical Observatory. We are grateful to the Chandra operations teams for performing the observations and providing software and calibration for the data analysis. We thank Keith Arnaud, for his continuing maintenance and development of XSPEC, and Gary Ferland and associates, for the maintenance and development of CLOUDY.

\section{REFERENCES}

Antonucci R., 1993, ARA\&A, 31, 473

Armentrout B. K., Kraemer S. B., Turner T. J., 2007, ApJ, 665, 237

Arnaud K. A., 1996, in Jacoby G. H., Barnes J., eds, ASP Conf. Ser. Vol. 101, Astronomical Data Analysis Software and Systems V. Astron. Soc. Pac., San Francisco, p. 17

Arzner K., Güdel M., Briggs K., Telleschi A., Schmidt M., Audard M., Scelsi L., Franciosini E., 2007, A\&A, 468, 501

Asplund M., Grevesse N., Sauval A., 2005, in Barnes T. G. I., Bash F. N., eds, ASP Conf. Ser. Vol. 336, Cosmic Abundances as Records of Stellar Evolution and Nucleosynthesis in honor of David L. Lambert. Astron. Soc. Pac., San Francisco, p. 25

Begelman M. C., 2004, in Ho L., ed., Carnegie Observatories Astrophysics Series Vol. 1, Coevolution of Black Holes and Galaxies, from the Carnegie Observatories Centennial Symposia. Cambridge Univ. Press, Cambridge, p. 374

Bentz M. C., Katz S., 2015, PASP, 127, 67

Bianchi S., Guainazzi M., Chiaberge M., 2006, A\&A, 448, 499

Bianchi S., Chiaberge M., Evans D. A., Guainazzi M., Baldi R. D., Matt G., Piconcelli E., 2010, MNRAS, 405, 553

Blandford R. D., Payne D. G., 1982, MNRAS, 199, 883
Bogdán A., Kraft R. P., Evans D. A., Andrade-Santos F., Forman W. R., 2017, ApJ, 848, 61

Canizares C. R. et al., 2005, PASP, 117, 1144

Collin S., Kawaguchi T., Peterson B. M., Vestergaard M., 2006, A\&A, 456, 75

Collins N. R., Kraemer S. B., Crenshaw D. M., Bruhweiler F. C., Meléndez M., 2009, ApJ, 694, 765

Couto J. D., Kraemer S. B., Turner T. J., Crenshaw D. M., 2016, ApJ, 833, 191

Cox A. N., 2000, in Cox A. N., ed., Allen's Astrophysical Quantities, 4th edn., AIP Press/Springer, New York

Crenshaw D. M., Kraemer S. B., Boggess A., Maran S. P., Mushotzky R. F., Wu C.-C., 1999, ApJ, 516, 750

Crenshaw D. M., Schmitt H. R., Kraemer S. B., Mushotzky R. F., Dunn J. P., 2010, ApJ, 708, 419

Crenshaw D. M., Fischer T. C., Kraemer S. B., Schmitt H. R., 2015, ApJ, 799,83

Das V. et al., 2005, AJ, 130, 945

Das V., Crenshaw D. M., Kraemer S. B., 2007, ApJ, 656, 699

Dauser T., Garcia J., Wilms J., Böck M., Brenneman L. W., Falanga M., Fukumura K., Reynolds C. S., 2013, MNRAS, 430, 1694

de Vaucouleurs G., de Vaucouleurs A., Corwin Herold G. J., Buta R. J., Paturel G., Fouque P., 1991, Third Reference Catalogue of Bright Galaxies. Springer, New York, NY, p. 2091

Dickey J. M., Lockman F. J., 1990, ARA\&A, 28, 215

Edelson R. A. et al., 1996, ApJ, 470, 364

Ferland G. J. et al., 2013, RMxAA, 49, 137

Ferland G. J. et al., 2017, Rev. Mex. Astron. Astrofis., 53, 385

Fischer T. C., Crenshaw D. M., Kraemer S. B., Schmitt H. R., 2013, ApJS, 209,1

Fischer T. C. et al., 2017, ApJ, 834, 30

Fischer T. C. et al., 2018, ApJ, 856, 102

Ganguly R., Brotherton M. S., 2008, ApJ, 672, 102

García J., Dauser T., Reynolds C. S., Kallman T. R., McClintock J. E., Wilms J., Eikmann W., 2013, ApJ, 768, 146

Gebhardt K. et al., 2000, ApJ, 539, L13

George I. M., Turner T. J., Netzer H., Nandra K., Mushotzky R. F., Yaqoob T., 1998, ApJS, 114, 73

Gonzalez-Martin O., Acosta-Pulido J. A., Perez Garcia A. M., Ramos Almeida C., 2010, ApJ, 723, 1748

Hagino K., Odaka H., Done C., Gandhi P., Watanabe S., Sako M., Takahashi T., 2015, MNRAS, 446, 663

Hopkins P. F., Elvis M., 2010, MNRAS, 401, 7

Hutchings J. B. et al., 1998, ApJ, 492, L115

Ishibashi W., Fabian A. C., Maiolino R., 2018, MNRAS, 476, 512

Kaastra J. S., Mewe R., Liedahl D. A., Komossa S., Brinkman A. C., 2000 , A\&A, 354, L83

Kallman T. R., Evans D. A., Marshall H., Canizares C., Longinotti A., Nowak M., Schulz N., 2014, ApJ, 780, 121

Kashi A., Proga D., Nagamine K., Greene J., Barth A. J., 2013, ApJ, 778, 50

Kaspi S. et al., 2001, ApJ, 554, 216

King A., Pounds K., 2015, ARA\&A, 53, 115

Kinkhabwala A. et al., 2002, ApJ, 575, 732

Kraemer S. B., Crenshaw D. M., 2000, ApJ, 532, 256

Kraemer S. B., Crenshaw D. M., Hutchings J. B., Gull T. R., Kaiser M. E., Nelson C. H., Weistrop D., 2000, ApJ, 531, 278

Kraemer S. B. et al., 2005, ApJ, 633, 693

Kraemer S. B. et al., 2006, ApJS, 167, 161

Kraemer S. B., Schmitt H. R., Crenshaw D. M., 2008, ApJ, 679, 1128

Kraemer S. B., Trippe M. L., Crenshaw D. M., Meléndez M., Schmitt H. R., Fischer T. C., 2009, ApJ, 698, 106

Kraemer S. B., Schmitt H., Crenshaw M., Meléndez M., Turner J., Guainazzi M., Mushotzky R., 2011, ApJ, 727, 130

Kraemer S. B., Sharma N., Turner T. J., George I. M., Crenshaw D. M., 2015, ApJ, 798, 53

Kraemer S. B., Tombesi F., Bottorff M. C., 2018, ApJ, 852, 35

Liedahl D. A., Paerels F., 1996, ApJ, 468, L33 
Maksym W. P. et al., 2019, ApJ, 872, 94

Netzer H., 1993, ApJ, 411, 594

Netzer H., 2015, ARA\&A, 53, 365

O’Dell S. L., Swartz D. A., Tice N. W., Plucinsky P. P., Grant C. E., Marshall H. L., Vikhlinin A., Tennant A. F., 2013, in Siegmund O. H., ed., Proc SPIE Conf. Ser. Vol. 8859, UV, X-Ray, and Gamma-Ray Space Instrumentation for Astronomy XVIII. SPIE, Bellingham, p. 88590F

Ogle P. M., Marshall H. L., Lee J. C., Canizares C. R., 2000, ApJ, 545, L81

Osterbrock D. E., Ferland G. J., 2006, in Osterbrock D. E., Ferland G. J., eds, Astrophysics of Gaseous Nebulae and Active Galactic Nuclei. 2nd edn., University Science Books, Sausalito, CA

Peterson B. M., 1997, An Introduction to Active Galactic Nuclei. Cambridge Univ. Press, Cambridge, New York

Porter R. L., Ferland G. J., Kraemer S. B., Armentrout B. K., Arnaud K. A., Turner T. J., 2006, PASP, 118, 920

Rees M. J., 1987, MNRAS, 228, 47P

Revalski M., Crenshaw D. M., Kraemer S. B., Fischer T. C., Schmitt H. R., Machuca C., 2018a, ApJ, 856, 46

Revalski M. et al., 2018b, ApJ, 867, 88

Reynolds C. S., 1997, MNRAS, 286, 513

Riffel R. A., Storchi-Bergmann T., Winge C., 2013, MNRAS, 430, 2249

Sako M., Kahn S. M., Paerels F., Liedahl D. A., 2000, ApJ, 543, L115

Storchi-Bergmann T., Lopes R. D. S., McGregor P. J., Riffel R. A., Beck T., Martini P., 2010, MNRAS, 402, 819

Tombesi F., Cappi M., Reeves J. N., Palumbo G. G. C., Braito V., Dadina M., 2011, ApJ, 742, 44

Turner T. J., George I. M., Nandra K., Mushotzky R. F., 1997, ApJS, 113, 23

Turner T. J., Kraemer S. B., Mushotzky R. F., George I. M., Gabel J. R., 2003, ApJ, 594, 128

Turnshek D. A., 1984, ApJ, 280, 51

Wang J. et al., 2011a, ApJ, 729, 75

Wang J., Fabbiano G., Elvis M., Risaliti G., Mundell C. G., Karovska M., Zezas A., 2011b, ApJ, 736, 62

Wang J. et al., 2011c, ApJ, 742, 23

Young A. J., Wilson A. S., Shopbell P. L., 2001, ApJ, 556, 6

\section{APPENDIX A.}

As noted in Section 2.2, our analysis was done with the data binned to a minimum of 10 counts per spectral bin. In order to determine if our binning affected the results, we performed a test for which we rebinned the data to a minimum of 20 counts per spectral bin.
Table A1. Comparison of fit parameters for the HETG data.

\begin{tabular}{|c|c|c|}
\hline $\begin{array}{l}\text { ATABLES } \\
\text { Zone }\end{array}$ & & $\begin{array}{l}\text { Velocity } \\
\left(\mathrm{km} \mathrm{s}^{-1}\right)\end{array}$ \\
\hline $\begin{array}{l}\text { HIGHION } 10 \text { counts bin } \\
\text { HIGHION } 20 \text { counts bin } \\
\text { MEDION } 10 \text { counts bin } \\
\text { MEDION } 20 \text { counts bin } \\
\text { NE }\end{array}$ & & $\begin{aligned}-369 & \pm 37 \\
-226 & \pm 43 \\
76 & \pm 45 \\
4 & \pm 43\end{aligned}$ \\
\hline $\begin{array}{l}\text { MTABLES } \\
\text { Zone }\end{array}$ & $\begin{array}{l}\log N_{\mathrm{H}} \\
\left(\mathrm{cm}^{-2}\right)\end{array}$ & Norm $^{a}$ \\
\hline $\begin{array}{l}\text { Zone } 1,10 \text { counts bin } \\
\text { 2one } 1,20 \text { counts bin }\end{array}$ & $\begin{array}{l}22.7 \pm 0.02 \\
22.7 \pm 0.02\end{array}$ & $\begin{array}{l}5.35( \pm 0.22) \mathrm{e}-02 \\
5.36( \pm 0.20) \mathrm{e}-02\end{array}$ \\
\hline $\begin{array}{l}\text { Reflection } \\
\text { model }\end{array}$ & $\log X i$ & Norm $^{a}$ \\
\hline $\begin{array}{l}\text { XILLVEREC, } 10 \\
\text { counts bin }\end{array}$ & $1.06 \pm 0.05$ & $1.18( \pm 0.10) \mathrm{e}-04$ \\
\hline $\begin{array}{l}\text { XILLVEREC, } 20 \\
\text { counts bin }{ }^{-1}\end{array}$ & $1.06 \pm 0.06$ & $1.18( \pm 0.11) \mathrm{e}-04$ \\
\hline
\end{tabular}

${ }^{a}$ Normalization of the model components during the XSPEC fitting.

We refitted the combined HEG and MEG spectra, allowing key parameters for the MTABLES, ATABLES, and the reflection model, XILLVEREC, to vary. The values that varied, compared to those for the 10 counts bin ${ }^{-1}$ fitting, are listed in Table A1. Other than some shifts in velocity, which would be expected as emission features spread out with the coarser binning, the results are essentially unaffected.

To test whether the significance of the emission features had changed, we reran the sliding Gaussian test described in Section 3.1. The results are shown in Fig. A1. As anticipated (see Section 2.2), several weak features are weaker or undetected in the 20 count binned spectra. Specifically, in the HEG, NeX $\alpha$ and the O VIII $\mathrm{RRC}$ are weaker, while in the MEG, the $\mathrm{Si} \mathrm{K}, \mathrm{Mg} \mathrm{XI} \beta$, and $\mathrm{Ne}$ IX $\beta$ are undetected, although the weakness of the $\mathrm{Si} \mathrm{K}$ is due to the subtraction of the XILLVEREC component. However, none of these features were used to constrain the ATABLES or photoionization models.
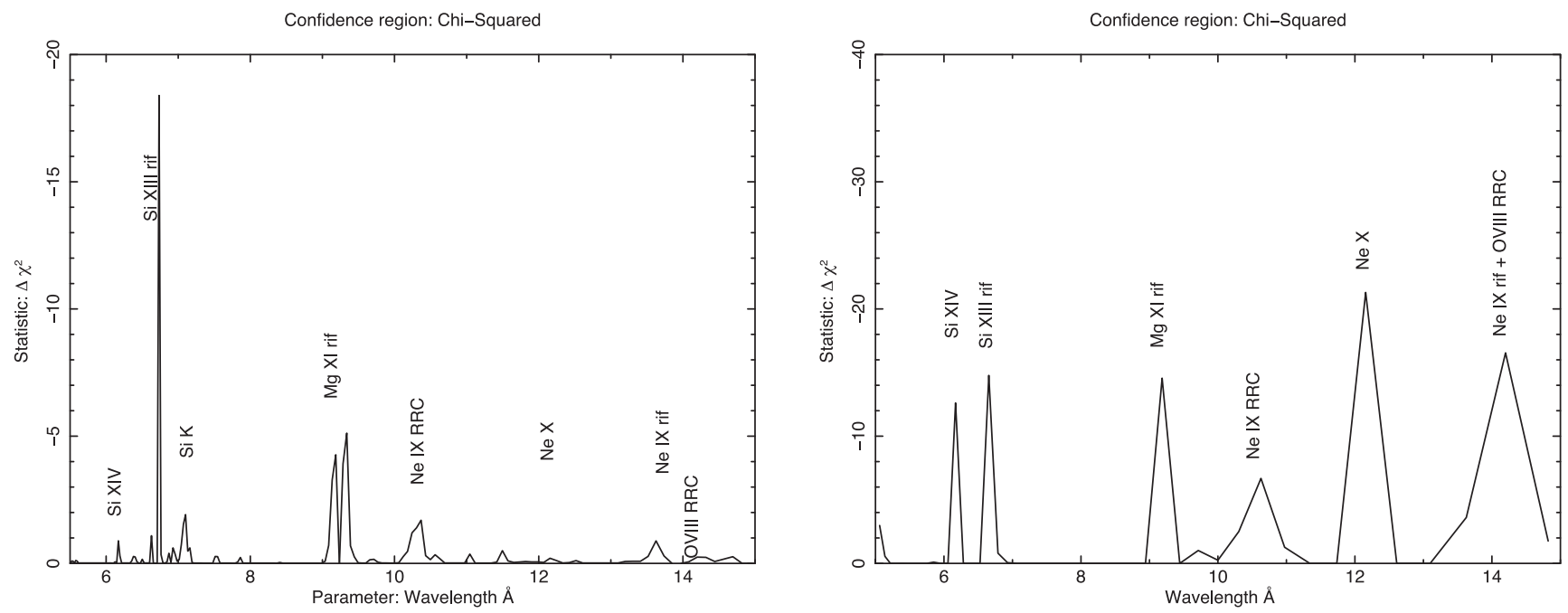

Figure A1. Sliding Gaussians tests, over the range 5.5-15 $\AA$, for the HEG (left-hand panel) and MEG (right-hand panel) spectra, for a minimum of 20 counts per spectral bin. The test was performed on the spectra with the inclusion of the absorbed power-law and reflection components, refitted for 20 counts bin ${ }^{-1}$ (see Table A1). The detected emission features are labelled. 
Two other differences highlight problems with the coarser binning. First, the Si XIII triplet is significantly stronger in the 20 counts bin ${ }^{-1}$ test. This is due to the inclusion of the unmodelled emission, discussed in Section 3.2, in the same bin as the Si XIII lines. The more significant difference is the absence of the $\mathrm{Mg}$ XII $\alpha$ line in either the HEG or MEG tests. The line is clearly present in the spectra, as shown in Fig. 1, however, it is at a minimum point in the continuum, hence the flux at this wavelength is dominated by the line emission. In the 20 count binning, the line flux is spread out in the bin, which makes it appear as continuum emission in the sliding Gaussian test. Nevertheless, Si XIV $\alpha$ and $\operatorname{NeX} \alpha$, the line diagnostics for the component in which the $\mathrm{Mg}$ XII forms, HIGHION, are detected by the sliding Gaussian. Furthermore, the photoionization models provide a good fit for $\mathrm{Mg}$ XII $\alpha$, as shown in Table 4 and Fig. 6. Therefore, we believe that the detection of $\operatorname{Mg}$ XII $\alpha$ in the 10 counts bin $^{-1}$ analysis is reliable.

In summary, the choice of a minimum of 10 counts bin ${ }^{-1}$ did not cause significant unreliability in our analysis. Furthermore, the 20 count binning introduced some problems in line detections, due to the smearing out of features or the inclusion of surrounding continuum in the same spectral bins as the lines.

This paper has been typeset from a $\mathrm{TE}_{\mathrm{E}} / \mathrm{LT} \mathrm{E} \mathrm{X}$ file prepared by the author. 